\title{
SCALING FOR A RANDOM POLYMER
}

R. van der Hofstad

F. den Hollander

Mathematical Institute

University of Utrecht

P.O. Box 80.010

3508 TA Utrecht

The Netherlands

\begin{abstract}
Let $Q_{n}^{\beta}$ be the law of the $n$-step random walk on $\mathbb{Z}^{d}$ obtained by weighting simple random walk with a factor $e^{-\beta}$ for every self-intersection (Domb-Joyce model of 'soft polymers'). It was proved by Greven and den Hollander (1993) that in $d=1$ and for every $\beta \in(0, \infty)$ there exist $\theta^{*}(\beta) \in(0,1)$ and $\mu_{\beta}^{*} \in\left\{\mu \in l^{1}(\mathbb{N}):\|\mu\|_{l^{1}}=1, \mu>0\right\}$ such that under the law $Q_{n}^{\beta}$ as $n \rightarrow \infty$ :

(i) $\theta^{*}(\beta)$ is the limit empirical speed of the random walk;

(ii) $\mu_{\beta}^{*}$ is the limit empirical distribution of the local times.

A representation was given for $\theta^{*}(\beta)$ and $\mu_{\beta}^{*}$ in terms of a largest eigenvalue problem for a certain family of $\mathbb{N} \times \mathbb{N}$ matrices. In the present paper we use this representation to prove the following scaling result as $\beta \downarrow 0$ :

(i) $\beta^{-\frac{1}{3}} \theta^{*}(\beta) \rightarrow b^{*}$;

(ii) $\beta^{-\frac{1}{3}} \mu_{\beta}^{*}\left(\left\lceil\cdot \beta^{-\frac{1}{3}}\right\rceil\right) \rightarrow^{L^{1}} \eta^{*}(\cdot)$.

The limits $b^{*} \in(0, \infty)$ and $\eta^{*} \in\left\{\eta \in L^{1}\left(\mathbb{R}^{+}\right):\|\eta\|_{L^{1}}=1, \eta>0\right\}$ are identified in terms of a Sturm-Liouville problem, which turns out to have several interesting properties.

The techniques that are used in the proof are functional analytic and revolve around the notion of epi-convergence of functionals on $L^{2}\left(\mathbb{R}^{+}\right)$. Our scaling result shows that the speed of soft polymers in $d=1$ is not right differentiable at $\beta=0$, which precludes expansion techniques that have been used successfully in $d \geq 5$ (Hara and Slade (1992a,b)) In simulations the scaling limit is seen for $\beta \leq 10^{-2}$.
\end{abstract}

Keywords: Random walk with self-repellence, weak interaction scaling limit, epi-convergence, Sturm-Liouville problem.

1991 Mathematical Subject Classification: primary 60J15; secondary 34B24, 47A55, 58E30.

Date: 4-7-1994. 


\section{o Introduction and main results}

\subsection{Model and motivation}

A polymer is a long chain of molecules with two characteristic properties: (i) an irregular shape (due to entanglement); (ii) a certain stiffness (due to sterical hindrance). One way of describing such a polymer is the following model, which is based on a random walk with self-repellence.

Let $\left(S_{i}\right)_{i>0}$ be simple random walk on $\mathbb{Z}^{d}(d \geq 1)$, starting at the origin. Let $P_{n}$ be its law on $n$-step paths and let $E_{P_{n}}$ be expectation w.r.t. $P_{n}$. Define a new law $Q_{n}^{\beta}$ on $n$-step paths by setting

$$
\frac{d Q_{n}^{\beta}}{d P_{n}}\left(\left(S_{i}\right)_{i=0}^{n}\right)=\frac{1}{Z_{n}^{\beta}} \exp \left[\Leftrightarrow \beta \sum_{\substack{i, j=0 \\ i \neq j}}^{n} 1\left\{S_{i}=S_{j}\right\}\right],
$$

where $Z_{n}^{\beta}$ is the normalizing constant

$$
Z_{n}^{\beta}=E_{P_{n}}\left(\exp \left[\Leftrightarrow \beta \sum_{\substack{i, j=0 \\ i \neq j}}^{n} 1\left\{S_{i}=S_{j}\right\}\right]\right)
$$

and $\beta \in[0, \infty]$ is a parameter. The law $Q_{n}^{\beta}$ is called the $n$-polymer measure with strength of repellence $\beta^{1}$.

Eqs.(0.1-2) define what is called the Domb-Joyce model of 'soft polymers', where the weight factor gives a penalty $e^{-\beta}$ for every self-intersection. The limiting cases $\beta=0$ and $\beta=\infty$ correspond to simple random walk resp. self-avoiding random walk. For a recent guide to the literature on this model the reader is referred to Madras and Slade (1993) Section 10.1.

It is generally believed that for $\beta \in(0, \infty]$ the mean square displacement behaves like

$$
E_{Q_{n}^{\beta}}\left[\left|S_{n}\right|^{2}\right] \sim D n^{2 \nu} \quad(n \rightarrow \infty),
$$

where $D=D(\beta, d)>0$ is some amplitude and $\nu=\nu(d)$ is a critical exponent. The latter is believed to be independent of $\beta$ and to assume the values ${ }^{2}$

$$
\begin{aligned}
\nu & =1 & & d=1 \\
& =\frac{3}{4} & & d=2 \\
& =0.588 \ldots & & d=3 \\
& =\frac{1}{2} & & d \geq 4 .
\end{aligned}
$$

Note that $\nu=\frac{1}{2}$ is the exponent for simple random walk $(\beta=0)$ in any $d \geq 1$ (with $D=1$ ). Apparently, the repellence changes the qualitative behavior when $d \leq 3$ but not when $d \geq 4^{3}$. The fact that $\nu$ is the same for all $\beta \in(0, \infty]$ says that soft polymers are in the same universality class as self-avoiding walk.

\footnotetext{
${ }^{1}$ Note that if $\beta>0$ then $\left(Q_{n}^{\beta}\right)_{n \geq 0}$ is not a consistent family, i.e., $Q_{n}^{\beta}$ is not the projection on $n$-step paths of the law of some process evolving in time (like $P_{n}$ ).

${ }^{2}$ The value in $d=3$ is well below $\max \left\{\frac{3}{d+2}, \frac{1}{2}\right\}$, the so-called Flory value (Madras and Slade (1993) Section 2.2).

${ }^{3}$ Actually, $d=4$ is a critical dimension where it is believed that $E_{Q_{n}^{\beta}}\left[\left|S_{n}\right|^{2}\right] \sim D n(\log n)^{\frac{1}{4}}$, containing a logarithmic correction to $(0.3-4)$.
} 
Sofar a rigorous proof of (0.3-4) has only been given for $d \geq 5$ (Hara and Slade (1992a,b) ${ }^{4}$ ) and for $d=1$ (Greven and den Hollander (1993)). In the latter work there is also a recipe for evaluating the amplitute $D(\beta, 1)$ as a function of $\beta$, which we next describe.

\subsection{Speed and local times in $d=1$}

Define the random variables

$$
\begin{aligned}
\theta_{n} & =\frac{1}{n}\left|S_{n}\right| \\
\mu_{n} & =\frac{1}{\left|R_{n}\right|} \sum_{x \in R_{n}} \delta_{\ell_{n}(x)},
\end{aligned}
$$

where

$$
\begin{aligned}
R_{n} & =\left(\min _{0 \leq i \leq n} S_{i}, \max _{0 \leq i \leq n} S_{i}\right) \cap \mathbb{Z} \\
\ell_{n}(x) & =\#\left\{0 \leq i<n: S_{i}=x\right\} .
\end{aligned}
$$

In words, $\theta_{n}$ is the empirical speed and $\mu_{n}$ is the empirical distribution of local times after $n$ steps. Theorems 1-3 below are taken from Greven and den Hollander (1993) and are the starting point of the present paper.

Theorem 1 For every $\beta \in(0, \infty)$ there exists $\theta^{*}(\beta) \in(0,1)$ such that

$$
\lim _{n \rightarrow \infty} Q_{n}^{\beta}\left(\left|\theta_{n} \Leftrightarrow \theta^{*}(\beta)\right| \leq \epsilon\right)=1 \text { for every } \epsilon>0,
$$

with $\beta \rightarrow \theta^{*}(\beta)$ analytic, $\lim _{\beta \downarrow 0} \theta^{*}(\beta)=0$ and $\lim _{\beta \rightarrow \infty} \theta^{*}(\beta)=1^{5}$.

Theorem 2 For every $\beta \in(0, \infty)$ there exists $\mu_{\beta}^{*} \in\left\{\mu \in l^{1}(\mathbb{N}):\|\mu\|_{l^{1}}=1, \mu>0\right\}$ such that

$$
\lim _{n \rightarrow \infty} Q_{n}^{\beta}\left(\left\|\mu_{n} \Leftrightarrow \mu_{\beta}^{*}\right\|_{l^{1}} \leq \epsilon\right)=1 \text { for every } \epsilon>0,
$$

with $\beta \rightarrow \mu_{\beta}^{*}$ analytic, $\lim _{\beta \downarrow 0} \mu_{\beta}^{*}=0$ and $\lim _{\beta \rightarrow \infty} \mu_{\beta}^{*}=\delta_{1}$ pointwise.

The limits $\theta^{*}(\beta)$ and $\mu_{\beta}^{*}$ in Theorems 1 and 2 can be found in terms of the following largest eigenvalue problem. Let $A_{r, \beta} \quad(r \in \mathbb{R}, \beta>0)$ be the matrix

$$
A_{r, \beta}(i, j)=e^{r(i+j-1)-\beta(i+j-1)^{2}} P(i, j)(i, j \in \mathbb{N}),
$$

where $P$ is the Markov matrix

$$
P(i, j)=\left(\begin{array}{c}
i+j \Leftrightarrow 2 \\
i \Leftrightarrow 1
\end{array}\right)\left(\frac{1}{2}\right)^{i+j-1} .
$$

\footnotetext{
${ }^{4}$ The proof in Hara and Slade $(1992 \mathrm{a}, \mathrm{b})$ is for $\beta=\infty$. However, the technique that is used (the so-called 'lace expansion') easily implies the same result for all $\beta \in(0, \infty]$. Brydges and Spencer (1985) earlier used the same technique to prove $(0.3-4)$ for $d \geq 5$ and $\beta$ sufficiently small.

${ }^{5}$ Note that $(0.5)$ and $(0.8)$ imply $(0.3)$ with $\nu(1)=1$ and $D(\beta, 1)=\left[\theta^{*}(\beta)\right]^{2}$.
} 
Let $\left(\lambda(r, \beta), \tau_{r, \beta}\right)$ be the unique solution of the largest eigenvalue problem ${ }^{6}$

$$
\begin{aligned}
& A_{r, \beta} \tau=\lambda \tau\left(\lambda>0, \tau \in l^{2}(\mathbb{N})\right) \\
& \|\tau\|_{l^{2}}=1, \tau>0
\end{aligned}
$$

Theorem 3 Fix $\beta \in(0, \infty)$. Let $r^{*}(\beta) \in(0, \infty)$ be the unique solution of

$$
\lambda(r, \beta)=1 .
$$

Then

$$
\begin{aligned}
\frac{1}{\theta^{*}(\beta)} & =\left[\frac{\partial}{\partial r} \lambda(r, \beta)\right]_{r=r^{*}(\beta)} \\
\mu_{\beta}^{*}(k) & =\left[\sum_{\substack{i, j \in \mathbb{N} \\
i+j-1=k}} \tau_{r, \beta}(i) A_{r, \beta}(i, j) \tau_{r, \beta}(j)\right]_{r=r^{*}(\beta)}(k \in \mathbb{N}) .
\end{aligned}
$$

The representation in Theorem 3 is not easy to manipulate, which is why precise analytical estimates of $\theta^{*}(\beta)$ and $\mu_{\beta}^{*}$ are hard to get. For instance, the intuitively appealing conjecture that $\beta \rightarrow \theta^{*}(\beta)$ is increasing still remains open (see Greven and den Hollander (1993)). However, it is easy to get numerical estimates (see section 0.3 ). Moreover, we shall see that (0.13-14) provide a good starting point for carrying out a scaling analysis as $\beta \downarrow 0$ (see sections $0.4-5)$, which is the main topic of the present paper.

\section{$0.3 \quad$ Numerical estimates of $r^{*}(\beta)$ and $\theta^{*}(\beta)$}

Table 1 below lists some numerical estimates of $r^{*}(\beta)$ and $\theta^{*}(\beta)$ obtained from (0.13-14), based on a $300 \times 300$ truncation of $A_{r, \beta}$ defined in $(0.10)$. We have used a standard iteration method to estimate the largest eigenvalue and corresponding eigenvector for a range of $r, \beta$-values.

$\begin{array}{lll}\beta & \beta^{-\frac{2}{3}} r^{*}(\beta) & \beta^{-\frac{1}{3}} \theta^{*}(\beta) \\ 2 & 1.696 & 0.793 \\ 0.5 & 1.730 & 1.055 \\ 10^{-2} & 2.011 & 1.10938 \\ 10^{-3} & 2.098 & 1.10930 \\ 10^{-4} & 2.144 & 1.10886 \\ 10^{-5} & 2.168 & 1.10910 \\ 10^{-6} & 2.179 & 1.10924\end{array}$

Table 1

There is ample evidence for the asymptotic behavior $r^{*}(\beta) \sim a^{*} \beta^{\frac{2}{3}}$ and $\theta^{*}(\beta) \sim b^{*} \beta^{\frac{1}{3}}(\beta \downarrow 0)$, with estimates $a^{*}=2.19 \pm 0.01$ and $b^{*}=1.109 \pm 0.001$.

\footnotetext{
${ }^{6} A_{r, \beta}: l^{2}(\mathbb{N}) \longmapsto l^{2}(\mathbb{N})$ is positive, self-adjoint and compact for all $r \in \mathbb{R}, \beta>0$. Both $(r, \beta) \rightarrow \lambda(r, \beta)$ and $(r, \beta) \rightarrow \tau_{r, \beta}$ are analytic. Moreover, $r \rightarrow \lambda(r, \beta)$ is strictly increasing and log-convex, $\lambda(0, \beta)<1$ and $\lambda(\infty, \beta)=\infty$ for every $\beta>0$ (see Greven and den Hollander (1993)).
} 
The value of $\theta^{*}(\beta)$ has been computed by making use of the identity

$$
\begin{aligned}
\frac{1}{\theta^{*}(\beta)} & =\sum_{k \in \mathbb{N}} k \mu_{\beta}^{*}(k) \\
& =2\left[\sum_{i \in \mathbb{N}} i \tau_{r^{*}(\beta), \beta}^{2}(i)\right] \Leftrightarrow 1
\end{aligned}
$$

(Greven and den Hollander (1993)). Since $\tau_{r, \beta}$ is easier to estimate than $\frac{\partial}{\partial r} \lambda(r, \beta)$, the relation in $(0.15)$ allows for better accuracy than $(0.14)$.

\subsection{Main results}

The goal of this paper is to turn the numerical observations in section 0.3 into a mathematical statement. Our results are formulated in Theorems 4-7 below.

1. Our main scaling theorem reads:

Theorem 4 There exist $a^{*}, b^{*} \in(0, \infty)$ and $\eta^{*} \in\left\{\eta \in L^{1}\left(\mathbb{R}^{+}\right):\|\eta\|_{L^{1}}=1, \eta>0\right\}$ such that as $\beta \downarrow 0$

$$
\begin{array}{lll}
\beta^{-\frac{2}{3}} r^{*}(\beta) & \rightarrow & a^{*} \\
\beta^{-\frac{1}{3}} \theta^{*}(\beta) & \rightarrow & b^{*} \\
\beta^{-\frac{1}{3}} \mu_{\beta}^{*}\left(\left\lceil\cdot \beta^{-\frac{1}{3}}\right\rceil\right) & \rightarrow L^{L^{1}} & \eta^{*}(\cdot) .
\end{array}
$$

2. The limits $a^{*}, b^{*}$ and $\eta^{*}$ in Theorem 4 can be identified in terms of the following SturmLiouville problem. For $a \in \mathbb{R}$, let $\mathcal{L}^{a}$ be the differential operator defined by

$$
\left(\mathcal{L}^{a} x\right)(u)=\left(2 a u \Leftrightarrow 4 u^{2}\right) x(u)+x^{\prime}(u)+u x^{\prime \prime}(u) \quad\left(x \in C^{\infty}\left(\mathbb{R}^{+}\right)\right) .
$$

In section 5 we shall show that the largest eigenvalue problem

$$
\begin{aligned}
& \mathcal{L}^{a} x \quad=\rho x\left(\rho \in \mathbb{R}, x \in L^{2}\left(\mathbb{R}^{+}\right) \cap C^{\infty}\left(\mathbb{R}^{+}\right)\right) \\
& \text {(i) }\|x\|_{L^{2}}=1, x>0 \\
& \text { (ii) } \quad \int_{0}^{\infty}\left\{u^{2}[x(u)]^{2}+u\left[x^{\prime}(u)\right]^{2}\right\} d u<\infty
\end{aligned}
$$

has a unique solution $\left(x^{a}, \rho(a)\right)$ with the following properties:

(i) $\quad a \rightarrow \rho(a)$ is analytic, strictly increasing and strictly convex on $\mathbb{R}$

(ii) $\rho(0)<0, \lim _{a \uparrow \infty} \rho(a)=\infty$ and $\lim _{a \downarrow-\infty} \rho(a)=\Leftrightarrow \infty$

(iii) $a \rightarrow x^{a}$ is analytic as a map from $\mathbb{R}$ to $L^{2}\left(\mathbb{R}^{+}\right)$.

The main part of our analysis to prove Theorem 4 will revolve around the following theorem, which is proved in sections 2-5:

Theorem 5 Fix $a \in \mathbb{R}$. As $\beta \downarrow 0$

$$
\begin{aligned}
& \beta^{-\frac{1}{3}}\left[\lambda\left(a \beta^{\frac{2}{3}}, \beta\right) \Leftrightarrow 1\right] \rightarrow \rho(a) \\
& \beta^{-\frac{1}{6}} \tau_{a \beta^{\frac{2}{3}, \beta}}\left(\left\lceil\cdot \beta^{-\frac{1}{3}}\right\rceil\right) \rightarrow \rightarrow^{L^{2}} x^{a}(\cdot) .
\end{aligned}
$$

We shall show in section 6 that $(0.20)$ identifies the limits in Theorem 4 as follows: 
Theorem $6 a^{*}, b^{*}$ and $\eta^{*}$ are given by

$$
\begin{aligned}
& a^{*} \text { is the unique solution of } \rho(a)=0 \\
& \frac{1}{b^{*}}=\rho^{\prime}\left(a^{*}\right) \\
& \eta^{*}(\cdot)=\frac{1}{2}\left[x^{a^{*}}\left(\frac{1}{2} \cdot\right)\right]^{2} .
\end{aligned}
$$

3. The analysis in section 5 of the Sturm-Liouville problem will lead to the following additional properties:

Theorem 7 (i) $u \rightarrow x^{a^{*}}(u)$ is analytic and strictly decreasing on $\mathbb{R}_{0}^{+}=[0, \infty)$.

(ii) $u \rightarrow u \frac{d}{d u} x^{a^{*}}(u)$ is unimodal with a minimum at $u=\frac{1}{2} a^{*}$.

(iii)

$$
\lim _{u \rightarrow \infty} u^{-\frac{3}{2}} \log x^{a^{*}}(u)=\Leftrightarrow \frac{4}{3}
$$

(iv)

$$
\frac{1}{b^{*}}=2 \int_{0}^{\infty} u\left[x^{a^{*}}(u)\right]^{2} d u .
$$

Theorems 4-7 are proved in sections 2-6. Section 1 contains preparations.

Our result $\theta^{*}(\beta) \sim b^{*} \beta^{\frac{1}{3}}$ implies that the speed is not right-differentiable at $\beta=0$. Thus the limit of weak repellence cannot be treated by perturbation type arguments (i.e., by doing an expansion of $(0.1-2)$ for small $\beta)$.

\subsection{Numerical estimates of $a^{*}, b^{*}$ and $\eta^{*}$}

Let $y^{a, \rho}$ be the unique power series solution of $\mathcal{L}^{a} y=\rho y$ with $y^{a, \rho}(0)=1$. We shall see in section 5 that this power series has infinite radius of convergence and has coefficients which satisfy a simple recurrence relation (see $(5.23)$ below). Moreover, we shall see that:

(i) $\rho(a)$ is simple

(ii) $\mathcal{S}_{a}=\left\{\rho \in \mathbb{R}: y^{a, \rho} \in L^{2}\left(\mathbb{R}^{+}\right)\right\}$is a countable set which has $\rho(a)$ as a maximum

(iii) $\rho \notin \mathcal{S}_{a}: \lim _{u \rightarrow \infty} y^{a, \rho}(u)= \pm \infty$

(iv) $\rho \in \mathcal{S}_{a}, \rho \neq \rho(a): y^{a, \rho}(u)<0$ for some $u>0$

(v) $y^{a, \rho(a)}=x^{a}$, the monotone solution of $(0.18)$.

Properties $(i) \Leftrightarrow(v)$ give us a way to estimate $a^{*}$ and $x^{a^{*}}$. Namely, put $\rho=0$ and consider $y^{a, 0}$, the unique power series solution of $\mathcal{L}^{a} y=0(a \in \mathbb{R})$. Since $a^{*}$ is the unique value of $a$ for which $y^{a, 0} \in L^{2}\left(\mathbb{R}^{+}\right)$and $y^{a, 0} \geq 0$, we can vary $a$ and tune into $a^{*}$ by looking at the tail behavior and the sign of $y^{a, 0}$. It turns out that this method is very sensitive indeed and that $a^{*}$ can be estimated by $a^{*}=2.189 \pm 0.001$. For $a$ outside this interval it was found that either $y^{a, 0}(u)<0$ for some $u \in[0,3]$, or $u \rightarrow y^{a, 0}(u)$ not monotone on $u \in[0,3]$.

[Figure 1]

Figure 1 compares $x^{a^{*}}$ with the numerical estimates in section 0.3 . The solid line is $u \rightarrow y^{a, 0}(u) /\left\|y^{a, 0}\right\|_{L^{2}}$ for $a=2.189$. The dots are the values of $\beta^{-\frac{1}{6}} \tau_{r^{*}(\beta), \beta}\left(\left\lceil u \beta^{-\frac{1}{3}}\right\rceil\right)$ for $\beta=10^{-4}$ and $\left[u \beta^{-\frac{1}{3}}\right\rceil=1, \ldots, 64$. The agreement is excellent. (For $\beta=10^{-5}$ and $\beta=10^{-6}$ all dots were found to lie on the solid line within printing precision.) 
Pick $a=2.189$. Since $y^{a, 0}$ is an approximation of $x^{a^{*}}$, we can estimate $\frac{1}{b^{*}}$ by the integral $2 \int_{0}^{\infty} u\left[y^{a, 0}(u)\right]^{2} d u$ (recall $(0.23)$ ). However, we have only computed $y^{a, 0}(u)$ for $u \in[0,3]$ and it turns out that this is not enough to get a good estimate of $b^{*}$ up to the third decimal. A better way is to use $(0.15)$ and estimate

$$
\frac{1}{b^{*}} \approx 2 \beta^{\frac{2}{3}} \sum_{i \in \mathbb{N}} i\left[y^{a, 0}\left(i \beta^{\frac{1}{3}}\right)\right]^{2} \Leftrightarrow \beta^{\frac{1}{3}} .
$$

This gives $b^{*}=1.109 \pm 0.001$.

\subsection{The Edwards model}

Westwater (1984) studies Brownian motion on $\mathbb{R}$ with self-repellence, i.e., the Edwards model where $(0.1)$ is replaced by

$$
\frac{d \nu_{T}^{g}}{d \mu_{T}}\left(\left(W_{t}\right)_{0 \leq t \leq T}\right)=\frac{1}{Z_{T}^{g}} \exp \left[\Leftrightarrow g \int_{0}^{T} d s \int_{0}^{T} d t \delta\left(W_{s} \Leftrightarrow W_{t}\right)\right] .
$$

Here $\mu_{T}$ is the Wiener measure on Brownian motion paths $\left(W_{t}\right)_{0 \leq t \leq T}, \delta$ the Dirac-function, $g \in[0, \infty)$ the repellence parameter and $Z_{T}^{g}$ the normalizing constant. ${ }^{7}$ We give two properties showing that the Edwards model arises as the weak interaction limit of the DombJoyce model.

Property 1 For every $g \in[0, \infty)$

$$
Q_{n}^{g n^{-\frac{3}{2}}}\left(\left(n^{-\frac{1}{2}} S_{\lceil t n\rceil}\right)_{0 \leq t \leq 1} \in \cdot\right) \Rightarrow \nu_{1}^{g}\left(\left(W_{t}\right)_{0 \leq t \leq 1} \in \cdot\right) \text { as } n \rightarrow \infty .
$$

Proof. See Brydges and Slade (1994) Theorem 1.3. The double sum in (0.1) equals $\Leftrightarrow(n+1)+$ $\sum_{x} \ell_{n}^{2}(x)$ (recall $(0.7)$ ), of which the first term may be absorbed into the normalizing constant $Z_{n}^{\beta}$ in (0.2). The key point is that $n^{-\frac{3}{2}} \sum_{x} \ell_{n}^{2}(x)$ under the law $P_{n}$ converges to $\int_{\mathbb{R}} \hat{\ell}_{1}^{2}(x) d x$ under the law $\mu_{1}$ (recall footnote 7 ). This immediately implies $(0.26)$. The analogous for $T \neq 1$ is obvious.

Westwater (1984) proves the following result which is analogous to Theorems 1 and 3 :

For every $g \in[0, \infty)$

$$
\lim _{T \rightarrow \infty} \nu_{T}^{g}\left(\left|\frac{1}{T}\right| W_{T}\left|\Leftrightarrow \hat{\theta}^{*}(g)\right| \leq \epsilon\right)=1 \text { for every } \epsilon>0,
$$

where

$$
\hat{\theta}^{*}(g)=\left[\frac{\partial}{\partial \lambda} E(g, \lambda)\right]_{\lambda=0}
$$

with $E(g, \lambda)$ the smallest eigenvalue in $L^{2}\left(\mathbb{R}^{+}\right)$of the operator $\hat{\mathcal{L}}^{g, \lambda}$ given by

$$
\left(\hat{\mathcal{L}}^{g, \lambda} y\right)(v)=\left[g v^{2}+\lambda v^{-2} \Leftrightarrow \frac{1}{2} v^{-1}\left(\frac{d^{2}}{d v^{2}}+\frac{1}{4} v^{-2}\right) v^{-1}\right] y(v) .
$$

(The term between round brackets equals $v^{\frac{1}{2}} \Delta_{\text {rad }}^{(2)} v^{-\frac{1}{2}}$ with $\Delta_{\text {rad }}^{(2)}$ the 2-dimensional Laplace operator.)

\footnotetext{
${ }^{7}$ The double integral in $(0.25)$ should be read as $\int_{\mathbb{R}} \hat{\ell}_{T}^{2}(x) d x$, where $\hat{\ell}_{T}(x)=\int_{0}^{T} d t \delta\left(W_{t}-x\right)$ is the density of the occupation time measure w.r.t. Lebesgue measure.
} 
Property 2 For every $g \in[0, \infty)$

$$
\begin{aligned}
& E(g, 0)=a^{*} g^{\frac{2}{3}} \\
& {\left[\frac{\partial}{\partial \lambda} E(g, \lambda)\right]_{\lambda=0}=b^{*} g^{\frac{1}{3}},}
\end{aligned}
$$

with $a^{*}, b^{*}$ the same constants as in Theorems \& and 6 .

Proof. Take the eigenvalue problem

$$
\left(\hat{\mathcal{L}}^{g, \lambda} y\right)(v)=E(g, \lambda) y(v) .
$$

Substitute into (0.31) the following change of variables:

$$
\begin{aligned}
& y(v)=v^{\frac{1}{2}} x\left(\frac{1}{2} g^{\frac{1}{3}} v^{2}\right) \\
& u \quad=\frac{1}{2} g^{\frac{1}{3}} v^{2} .
\end{aligned}
$$

Then, after a small computation, we obtain the Sturm-Liouville problem in $(0.17-18)$

$$
\left(\mathcal{L}^{a} x\right)(u)=\rho x(u)
$$

with

$$
\begin{aligned}
& a=g^{-\frac{2}{3}} E(g, \lambda) \\
& \rho=g^{-\frac{1}{3}} \lambda .
\end{aligned}
$$

Think of (0.34) as a parametrization of the curve $a \rightarrow \rho(a)$ in terms of $\lambda$. Recalling the definition of $a^{*}, b^{*}$ in (0.21), we now get from (0.33-0.34) that

$$
\rho\left(a^{*}\right)=0 \Leftrightarrow a^{*}=g^{-\frac{2}{3}} E(g, 0)
$$

and

$$
\begin{aligned}
{\left[\frac{\partial}{\partial \lambda} E(g, \lambda)\right]_{\lambda=0} } & =g^{-\frac{1}{3}}\left[\frac{\partial}{\partial \rho} E\left(g, \rho g^{\frac{1}{3}}\right)\right]_{\rho=0} \\
& =g^{-\frac{1}{3}}\left[\frac{\partial}{\partial \rho}\left(a(\rho) g^{\frac{2}{3}}\right)\right]_{\rho=0} \\
& =g^{\frac{1}{3}} a^{\prime}(0) \\
& =g^{\frac{1}{3}} \frac{1}{\rho^{\prime}\left(a^{*}\right)} \\
& =g^{\frac{1}{3}} b^{*}
\end{aligned}
$$

where $\rho \rightarrow a(\rho)$ is the inverse function of $a \rightarrow \rho(a)$.

Properties 1 and 2 show that Theorems 4 and 6 connect up nicely with the Edwards model. 
We close this section with a heuristic explanation of the power $\frac{1}{3}$ in our result $\theta^{*}(\beta) \sim$ $b^{*} \beta^{\frac{1}{3}}(\beta \downarrow 0)$. First, by Brownian scaling

$$
E_{\nu_{1}^{g T} \frac{3}{2}}\left(W_{1}^{2}\right)=\frac{1}{T} E_{\nu_{T}^{g}}\left(W_{T}^{2}\right)
$$

Since, according to (0.27),

$$
\left[\hat{\theta}^{*}(g)\right]^{2}=\lim _{T \rightarrow \infty} \frac{1}{T^{2}} E_{\nu_{T}^{g}}\left(W_{T}^{2}\right),
$$

it follows that

$$
\hat{\theta}^{*}(g)=g^{\frac{1}{3}} \hat{\theta}^{*}(1)
$$

Next, according to Theorem 1 ,

$$
\left[\theta^{*}(g)\right]^{2}=\lim _{n \rightarrow \infty} \frac{1}{n^{2}} E_{Q_{n}^{g}}\left(S_{n}^{2}\right) .
$$

Moreover, by Property 1 we know that for $g, T$ fixed

$$
\frac{1}{n} E_{Q_{n}^{g\left(\frac{T}{n}\right)^{\frac{3}{2}}}}\left(S_{n}^{2}\right) \sim E_{\nu_{1}^{g T^{\frac{3}{2}}}}\left(W_{1}^{2}\right)(n \rightarrow \infty) .
$$

Now, if we assume that (0.41) continues to hold for $g$ fixed and $T=n$, then by using $(0.40-41)$ resp. (0.37-38) we arrive at

$$
\begin{aligned}
{\left[\theta^{*}(g)\right]^{2} } & \sim \frac{1}{n^{2}} E_{Q_{n}^{g}}\left(S_{n}^{2}\right) \\
& \sim E_{\nu_{1}^{g T^{\frac{3}{2}}}}\left(W_{1}^{2}\right) \\
& =\frac{1}{T^{2}} E_{\nu_{T}^{g}}\left(W_{T}^{2}\right) \\
& \sim\left[\hat{\theta}^{*}(g)\right]^{2}(T=n \rightarrow \infty) .
\end{aligned}
$$

The above argument has uniformity problems because $(0.39)$ and $(0.42)$ would imply $\theta^{*}(g)=g^{\frac{1}{3}} \theta^{*}(1)$ for all $g$. However, this cannot be true because $\theta^{*}(g) \leq 1$ for all $g$. Nevertheless, it explains the power $\frac{1}{3}$ without using the explicit solution.

\section{Preparations}

In this section we formulate the functional analytic framework in which we are going to approach our scaling theorem. Section 1.1 shows that our key result, Theorem 5 in section 0.4 , is equivalent to convergence of a variational problem involving a certain functional $F_{\beta}^{a}$ to a variational problem involving some limit functional $F^{a}$ (Lemma 1 and Proposition 1 below). Section 1.2 shows that this convergence holds when $F_{\beta}^{a}$ epi-converges to $F^{a}$ and certain compactness properties are satisfied (Proposition 2 below). In this section we also formulate the main steps that have to be checked in order to prove these facts (Proposition 3 below). In section 1.3 we collect some properties of the matrix $P$, defined in $(0.11)$, that will be needed in the proofs. 


\subsection{A variational representation}

Rayleigh's formula for the pair $\left(\lambda(r, \beta), \tau_{r, \beta}\right)$ defined in $(0.12)$ reads

$$
\begin{aligned}
& \text { (i) } \lambda(r, \beta)=\max _{y \in l^{2}(\mathbb{N}), y \geq 0}\|y\|_{l^{2}} \leq 1 \\
& \text { (ii) } \tau_{r, \beta} \text { is the unique maximizer. }
\end{aligned}
$$

In anticipation of the scaling suggested by Table 1 , we pick $r=a \beta^{\frac{2}{3}}(a \in \mathbb{R})$ and rewrite (1.1) in the following form. Define the functional $F_{\beta}^{a}: L^{2}\left(\mathbb{R}^{+}\right) \rightarrow \mathbb{R}$ as

$$
F_{\beta}^{a}(x)=\beta^{-\frac{2}{3}} \int_{0}^{\infty} d u \int_{0}^{\infty} d v x(u) x(v) A_{a \beta^{\frac{2}{3}, \beta}}\left(\left\lceil u \beta^{-\frac{1}{3}}\right\rceil,\left\lceil v \beta^{-\frac{1}{3}}\right\rceil\right) \Leftrightarrow \beta^{-\frac{1}{3}}\|x\|_{L^{2}}^{2} .
$$

Lemma 1 For all $\beta>0$

$$
\begin{aligned}
& \text { (i) } \beta^{-\frac{1}{3}}\left[\lambda\left(a \beta^{\frac{2}{3}}, \beta\right) \Leftrightarrow 1\right]=\max _{\substack{x \in L^{2}\left(\mathbb{R}^{+}\right), x \geq 0 \\
\|x\|_{L^{2}}=1}} F_{\beta}^{a}(x) \\
& \text { (ii) } \beta^{-\frac{1}{6}} \tau_{a \beta^{\frac{2}{3}, \beta}}\left(\left\lceil\cdot \beta^{-\frac{1}{3}}\right\rceil\right) \text { is the unique maximizer. }
\end{aligned}
$$

Proof. (i) Fix $\beta>0$. For $x \in L^{2}\left(\mathbb{R}^{+}\right)$define

$$
\hat{x}(i)=\beta^{-\frac{1}{6}} \int_{(i-1) \beta^{\frac{1}{3}}}^{i \beta^{\frac{1}{3}}} x(u) d u(i \in \mathbb{N}) .
$$

Then the first term in (1.2) equals $\beta^{-\frac{1}{3}}\left\langle\hat{x}, A_{a \beta^{\frac{2}{3}, \beta}} \hat{x}\right\rangle_{l^{2}}$. Hence using (1.1)(i) we may write

$$
\beta^{-\frac{1}{3}}\left[\lambda\left(a \beta^{\frac{2}{3}}, \beta\right) \Leftrightarrow 1\right]=\max _{\substack{y \in l^{2}(\mathbb{N}), y \geq 0, x \in L^{2}\left(\mathbb{R}^{+}\right), x \geq 0,\|y\|_{l^{2}} \leq 1}} F_{\beta}^{a}(x) .
$$

Note that, by Cauchy-Schwarz, we have $\|\hat{x}\|_{l^{2}} \leq\|x\|_{L^{2}}$ and so the restrictions $\|y\|_{l^{2}} \leq$ $1,\|x\|_{L^{2}}=1, \hat{x}=y$ in (1.5) are compatible. Interchange the two maxima in (1.5) to get the claim.

(ii) Use that $\|\hat{x}\|_{l^{2}}=\|x\|_{L^{2}}$ iff $x(u)=\beta^{-\frac{1}{6}} \hat{x}(i)$ for $u \in\left((i \Leftrightarrow 1) \beta^{\frac{1}{3}}, i \beta^{\frac{1}{3}}\right]$.

In sections 2-5 we shall prove:

Proposition 1 As $\beta \downarrow 0$

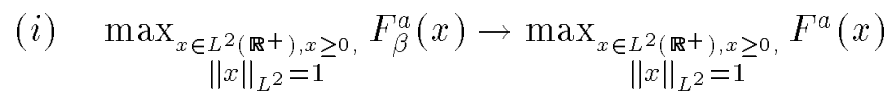

(ii) unique maximizer l.h.s. $\rightarrow^{L^{2}}$ unique maximizer r.h.s.,

where the limit functional $F^{a}: L^{2}\left(\mathbb{R}^{+}\right) \rightarrow \overline{\mathbb{R}}$ is given by

$$
F^{a}(x)=\int_{0}^{\infty}\left\{\left(2 a u \Leftrightarrow 4 u^{2}\right)[x(u)]^{2} \Leftrightarrow u\left[x^{\prime}(u)\right]^{2}\right\} d u,
$$

with the understanding that $F^{a}(x)=\Leftrightarrow \infty$ if the integral is not defined.

Note that $F^{a}(x)=\left\langle x, \mathcal{L}^{a} x\right\rangle_{L^{2}}$ for all $x$ where both sides are finite, with $\mathcal{L}^{a}$ as defined in $(0.17)$.

Lemma 1 and Proposition 1 imply Theorem 5. To prove Proposition 1, we shall need the notion of epi-convergence, which we next explain. 


\subsection{Epi-convergence}

Let $(X, \tau)$ be a metrizable topological space and let $Y \subset X$ be dense in $X$. Let

$$
\begin{aligned}
& G_{\beta}: X \rightarrow \mathbb{R}(\beta>0) \\
& G: X \rightarrow \overline{\mathbb{R}} .
\end{aligned}
$$

Definition 1 The family $\left(G_{\beta}\right)_{\beta>0}$ is said to be epi-convergent to $G$ on $Y$, written

$$
e \Leftrightarrow \lim _{\beta \downarrow 0} G_{\beta}=G \text { on } Y,
$$

if the following properties hold:

$$
\begin{aligned}
& \text { (i) } \forall x_{\beta} \rightarrow^{\tau} x \text { in } Y: \lim \sup _{\beta \downarrow 0} G_{\beta}\left(x_{\beta}\right) \leq G(x) \\
& \text { (ii) } \exists x_{\beta} \rightarrow^{\tau} x \text { in } Y: \lim \inf _{\beta \downarrow 0} G_{\beta}\left(x_{\beta}\right) \geq G(x) .
\end{aligned}
$$

The importance of the notion of epi-convergence is contained in the following proposition:

Proposition 2 Suppose that

(1) $e \Leftrightarrow \lim _{\beta \downarrow 0} G_{\beta}=G$ on $Y$

(2) $\forall \beta>0: G_{\beta}$ is continuous on $X$ and has a unique maximizer $\bar{x}_{\beta} \in X$

(3) $\exists K \subset Y$ such that

(i) $K$ is $\tau$-relatively compact in $X$

(ii) $G$ has a unique maximizer $\bar{x} \in \bar{K}$

(iii) $\exists\left(x_{\beta}\right)_{\beta>0} \subset \bar{K}$ such that $x_{\beta} \Leftrightarrow \bar{x}_{\beta} \rightarrow^{\tau} 0$ and $G_{\beta}\left(x_{\beta}\right) \Leftrightarrow G_{\beta}\left(\bar{x}_{\beta}\right) \rightarrow 0$ as $\beta \downarrow 0$.

Then as $\beta \downarrow 0$

$$
\begin{gathered}
\sup _{x \in X} G_{\beta}(x) \rightarrow \sup _{x \in X} G(x) \\
\bar{x}_{\beta} \rightarrow^{\tau} \bar{x} .
\end{gathered}
$$

Proof. See Attouch (1984) Theorem 1.10 and Proposition 1.14.

Remark: Epi-convergence differs from pointwise convergence: $\lim _{\beta \downarrow 0} G_{\beta}(x)=G(x)$ for all $x \in Y$. Namely, (1.10)(i),(ii) are weaker in the sense that they require only inequalities, but stronger in the sense that they involve limits in neighborhoods rather than single points. Epiconvergence is a unilateral notion. We have chosen the direction that is suitable for suprema rather than infima.

Fix $a \in \mathbb{R}$. We are going to apply Proposition 2 with the following choices:

$$
\begin{aligned}
X & =\left\{x \in L^{2}\left(\mathbb{R}^{+}\right): x \geq 0,\|x\|_{L^{2}}=1\right\} \\
Y & =X \cap C^{1}\left(\mathbb{R}_{0}^{+}\right) \\
\tau & =\text { topology induced by }\|\cdot\|_{L^{2}} \\
K & =K_{C}^{a}=\left\{x \in Y: F^{a}(x) \geq \Leftrightarrow C\right\} \\
G_{\beta} & =F_{\beta}^{a} \\
G & =F^{a}
\end{aligned}
$$

with $F_{\beta}^{a}$ and $F^{a}$ defined in $(1.2)$ and $(1.7)$ and with $C$ large enough so that $K_{C}^{a} \neq \emptyset$. Our main result is:

Proposition 3 Assumptions (1)-(3) in Proposition 2 hold for the choice in (1.13).

We prove Assumption (1) in section 2, (3)(i),(ii) in section 5 and (3)(iii) in section 3. We already know (2) to be true because of Lemma 1(ii).

Proposition 3 proves Proposition 1 in section 1.1. 


\subsection{Properties of $P$}

We list a few identities and estimates for the matrix $P$, defined in $(0.11)$, that will be needed later on.

Lemma 2 For every $i \geq 1, k \geq 0$

$$
\sum_{j \geq 1} \frac{(i+j+k \Leftrightarrow 2) !}{(i+j \Leftrightarrow 2) !} P(i, j)=2^{k} \frac{(i+k \Leftrightarrow 1) !}{(i \Leftrightarrow 1) !} .
$$

Proof. Elementary. Use that the summands in the 1.h.s. can be rewritten as $P(i+k, j)$ times the r.h.s. Then use that $\sum_{j \geq 1} P(i+k, j)=1$.

Lemma 3 (i) For $i, j \rightarrow \infty$ such that $i \Leftrightarrow j=o\left((i+j)^{\frac{2}{3}}\right)$

$$
P(i, j)=\left\{\frac{1}{\sqrt{2 \pi(i+j)}} \exp \left[\Leftrightarrow \frac{(i \Leftrightarrow j)^{2}}{2(i+j)}\right]\right\}\left[1+\mathcal{O}\left((i+j)^{-\frac{1}{3}}\right)\right] .
$$

(ii) There exist $0<c_{1}<c_{2}<\infty$ such that

$$
\exp \left[\Leftrightarrow c_{2} \frac{(i \Leftrightarrow j)^{2}}{(i+j)}\right] \leq P(i, j) \leq \exp \left[\Leftrightarrow c_{1} \frac{(i \Leftrightarrow j)^{2}}{(i+j)}\right] \text { for all } i, j \geq 1 \text {. }
$$

Proof. Via Stirling's formula. See also Révész (1990) Theorem 2.8.

Lemma 2 allows us to compute the following moments, which we shall need in section 2 :

$$
\begin{aligned}
& \sum_{j \geq 1}(i+j \Leftrightarrow 1)^{n} P(i, j)=2 i \quad(n=1) \\
& 8 i^{3}+12 i^{2}+6 i \quad(n=3) \\
& 16 i^{4}+48 i^{3}+72 i^{2}+32 i \quad(n=4) \text {. }
\end{aligned}
$$

Lemma 3(i) is a Gaussian approximation of $P$, while Lemma 3(ii) shows that $P(i, j)$ is small away from the diagonal.

Lemma 4 For all $i, j \geq 0$ with $(i, j) \neq(0,0)$

$$
P(i+1, j)+P(i, j+1) \Leftrightarrow 2 P(i+1, j+1)=0
$$

with the convention $P(i, 0)=P(0, j)=0$.

Proof. Elementary.

Lemma 4 will be needed in sections 2 and 3 to obtain monotonicity properties and estimates of $\tau_{a \beta^{\frac{2}{3}, \beta}}$, the eigenvector of $A_{a \beta^{\frac{2}{3}, \beta}}$. 


\section{$2 \quad\left(F_{\beta}^{a}\right)_{\beta>0}$ is epi-convergent to $F^{a}$}

In this section we prove Assumption (1) in Proposition 2 for the choice in (1.13).

This section is technically somewhat involved, as it consists of a chain of estimates and inequalities that are needed to handle the epi-convergence. The proof is contained in Lemmas 5-8 below. Throughout sections 2 and 3 we fix $a \in \mathbb{R}$ and we write the abbreviations $F_{\beta}=F_{\beta}^{a}$, $F=F^{a}, A_{\beta}=A_{a \beta^{\frac{2}{3}, \beta}}, \lambda(\beta)=\lambda\left(a \beta^{\frac{2}{3}}, \beta\right), \tau_{\beta}=\tau_{a \beta^{\frac{2}{3}, \beta}}$.

We begin by splitting $F_{\beta}, F$ into two parts, namely (recall (1.2) and (1.7))

$$
\begin{aligned}
& F_{\beta}=F_{\beta}^{1}+F_{\beta}^{2} \\
& F=F^{1}+F^{2}
\end{aligned}
$$

with

$$
\begin{aligned}
& F_{\beta}^{1}(x)=\beta^{-\frac{2}{3}} \int_{0}^{\infty} d u \int_{0}^{\infty} d v x^{2}(u)\left[A_{\beta} \Leftrightarrow P\right]\left(\left\lceil u \beta^{-\frac{1}{3}}\right\rceil,\left\lceil v \beta^{-\frac{1}{3}}\right\rceil\right) \\
& F_{\beta}^{2}(x)=\Leftrightarrow \frac{1}{2} \beta^{-\frac{2}{3}} \int_{0}^{\infty} d u \int_{0}^{\infty} d v[x(u) \Leftrightarrow x(v)]^{2} A_{\beta}\left(\left\lceil u \beta^{-\frac{1}{3}}\right\rceil,\left\lceil v \beta^{-\frac{1}{3}}\right\rceil\right)
\end{aligned}
$$

and

$$
\begin{aligned}
& F^{1}(x)=\int_{0}^{\infty} d u\left(2 a u \Leftrightarrow 4 u^{2}\right) x^{2}(u) \\
& F^{2}(x)=\Leftrightarrow \int_{0}^{\infty} d u u\left[x^{\prime}(u)\right]^{2} .
\end{aligned}
$$

Lemma $5 \forall x_{\beta} \rightarrow^{L^{2}} x$ in $X: \limsup _{\beta \downarrow 0} F_{\beta}^{1}\left(x_{\beta}\right) \leq F^{1}(x)$.

Proof. Abbreviate

$$
\epsilon_{\beta}(i, j)=a \beta^{\frac{2}{3}}(i+j \Leftrightarrow 1) \Leftrightarrow \beta(i+j \Leftrightarrow 1)^{2},
$$

which is the exponent appearing in $A_{\beta}(i, j)$, i.e., $A_{\beta}=e^{e_{\beta}} P$ (see $(0.10)$ ). We note that $e_{\beta}$ has the following properties:

$$
\begin{aligned}
& \text { (i) } e_{\beta}(i, j) \leq 0 \text { for } i \geq a \beta^{-\frac{1}{3}}, j \geq 1 \\
& \text { (ii) } e_{\beta}(i, j) \leq \frac{1}{4} a^{2} \beta^{\frac{1}{3}} \text { for } i, j \geq 1 .
\end{aligned}
$$

Hence, for small enough $\beta$ and large enough $N$

$$
\begin{aligned}
F_{\beta}^{1}\left(x_{\beta}\right) \leq & \beta^{-\frac{2}{3}} \int_{0}^{N} d u \int_{0}^{\infty} d v x_{\beta}^{2}(u) \\
& \times\left\{e_{\beta}\left(\left\lceil u \beta^{-\frac{1}{3}}\right\rceil,\left\lceil v \beta^{-\frac{1}{3}}\right\rceil\right)+e_{\beta}^{2}\left(\left\lceil u \beta^{-\frac{1}{3}}\right\rceil,\left\lceil v \beta^{-\frac{1}{3}}\right\rceil\right)\right\} P\left(\left\lceil u \beta^{-\frac{1}{3}}\right\rceil,\left\lceil v \beta^{-\frac{1}{3}}\right\rceil\right)
\end{aligned}
$$

(use that $e^{t} \leq 1+t+t^{2}$ for $t \ll 1$ and $t \leq 0$ ). The integral over $v$ can be transformed into the following sum:

$$
\beta^{\frac{1}{3}} \sum_{j \geq 1}\left\{e_{\beta}(i, j)+e_{\beta}^{2}(i, j)\right\} P(i, j) \text { with } i=\left\lceil u \beta^{-\frac{1}{3}}\right\rceil .
$$

Using (1.17), we can carry out the summation. Namely,

$$
\begin{aligned}
\sum_{j \geq 1} e_{\beta}(i, j) P(i, j)= & a \beta^{\frac{2}{3}}(2 i) \Leftrightarrow \beta\left(4 i^{2}+2 i\right) \\
\sum_{j \geq 1} e_{\beta}^{2}(i, j) P(i, j)= & a^{2} \beta^{\frac{4}{3}}\left(4 i^{2}+2 i\right) \Leftrightarrow 2 a \beta^{\frac{5}{3}}\left(8 i^{3}+12 i^{2}+6 i\right) \\
& +\beta^{2}\left(16 i^{4}+48 i^{3}+72 i^{2}+32 i\right) .
\end{aligned}
$$


Since $i=\left\lceil u \beta^{-\frac{1}{3}}\right\rceil \leq(N+1) \beta^{-\frac{1}{3}}$, the contribution to (2.6) of the second sum can be estimated above by

$$
\beta^{\frac{1}{3}}\left(6 a^{2}(N+1)^{2}+168(N+1)^{4}\right) \int_{0}^{N} d u x_{\beta}^{2}(u)=\mathcal{O}\left(\beta^{\frac{1}{3}}\right),
$$

where we use that $\left\|x_{\beta}\right\|_{L^{2}}=1$. The error term is uniform in $x_{\beta}$ for fixed $N$. Hence we get

$$
\begin{aligned}
F_{\beta}^{1}\left(x_{\beta}\right) \leq & \beta^{-\frac{1}{3}} \int_{0}^{N} d u x_{\beta}^{2}(u) \\
& \times\left\{a \beta^{\frac{2}{3}}\left(2\left\lceil u \beta^{-\frac{1}{3}}\right\rceil\right) \Leftrightarrow \beta\left(4\left\lceil u \beta^{-\frac{1}{3}}\right\rceil^{2}+2\left\lceil u \beta^{-\frac{1}{3}}\right\rceil\right)\right\}+\mathcal{O}\left(\beta^{\frac{1}{3}}\right) \\
= & \int_{0}^{N} d u x_{\beta}^{2}(u)\left(2 a u \Leftrightarrow 4 u^{2}\right)+\mathcal{O}\left(\beta^{\frac{1}{3}}\right) .
\end{aligned}
$$

Now let $\beta \downarrow 0$. Then we obtain, recalling that $x_{\beta} \rightarrow^{L^{2}} x$,

$$
\begin{aligned}
\lim \sup _{\beta \downarrow 0} F_{\beta}^{1}\left(x_{\beta}\right) & \leq \lim \sup _{\beta \downarrow 0} \int_{0}^{N} d u x_{\beta}^{2}(u)\left(2 a u \Leftrightarrow 4 u^{2}\right) \\
& =\int_{0}^{N} d u x^{2}(u)\left(2 a u \Leftrightarrow 4 u^{2}\right) .
\end{aligned}
$$

Finally, let $N \rightarrow \infty$ and note that the r.h.s. of (2.11) converges to $F^{1}(x)$.

Lemma $6 \forall x \in X: \liminf _{\beta \downarrow 0} F_{\beta}^{1}(x) \geq F^{1}(x)$.

Proof. Estimate

$$
F_{\beta}^{1}(x) \geq \beta^{-\frac{2}{3}} \int_{0}^{\infty} d u \int_{0}^{\infty} d v x^{2}(u) e_{\beta}\left(\left\lceil u \beta^{-\frac{1}{3}}\right\rceil,\left\lceil v \beta^{-\frac{1}{3}}\right\rceil\right) P\left(\left\lceil u \beta^{-\frac{1}{3}}\right\rceil,\left\lceil v \beta^{-\frac{1}{3}}\right\rceil\right)
$$

(use that $e^{t} \geq 1+t$ for all $t$ ). The integral over $v$ is $\beta^{\frac{1}{3}}$ times the first sum computed in (2.8) with $i=\left\lceil u \beta^{-\frac{1}{3}}\right\rceil$. Hence

$$
\begin{aligned}
F_{\beta}^{1}(x) \geq & \beta^{-\frac{1}{3}} \int_{0}^{\infty} d u x^{2}(u) \\
& \times\left\{a \beta^{\frac{2}{3}}\left(2 u \beta^{-\frac{1}{3}}\right) \Leftrightarrow \beta\left(4\left(u \beta^{-\frac{1}{3}}+1\right)^{2}+2\left(u \beta^{-\frac{1}{3}}+1\right)\right)\right\} \\
= & \int_{0}^{\infty} d u x^{2}(u)\left(2 a u \Leftrightarrow 4 u^{2}\right)+\mathcal{O}\left(\beta^{\frac{1}{3}}\right) .
\end{aligned}
$$

Now let $\beta \downarrow 0$. Then the claim follows.

Lemma $7 \forall x_{\beta} \rightarrow^{L^{2}} x$ in $X$ with $x \in Y: \lim \sup _{\beta \downarrow 0} F_{\beta}^{2}\left(x_{\beta}\right) \leq F^{2}(x)$.

Proof. The proof is in Steps 1-3 below.

STEP 1 For every $\epsilon>0$ and $N, M$ finite

$$
F_{\beta}^{2}\left(x_{\beta}\right) \leq \Leftrightarrow \frac{1}{2}\left(1+\mathcal{O}\left(\beta^{\frac{1}{9}}\right)\right) \int_{\epsilon}^{N} d u \int_{-M}^{M} d w\left[\frac{1}{\beta^{\frac{1}{6}}}\left\{x_{\beta}(u) \Leftrightarrow x_{\beta}\left(u+w \beta^{\frac{1}{6}}\right)\right\}\right]^{2} N_{2 u}(w),
$$

where $N_{2 u}$ is the Gaussian with mean zero and variance $2 u$. 
Proof. Pick $\epsilon>0$ and $N, M$ finite. Then

$$
F_{\beta}^{2}\left(x_{\beta}\right) \leq \Leftrightarrow \frac{1}{2} \beta^{-\frac{2}{3}} e^{-9 N^{2} \beta^{\frac{1}{3}}} \int_{\epsilon}^{N} d u \int_{u-M \beta^{\frac{1}{6}}}^{u+M \beta^{\frac{1}{6}}} d v\left[x_{\beta}(u) \Leftrightarrow x_{\beta}(v)\right]^{2} P\left(\left\lceil u \beta^{-\frac{1}{3}}\right\rceil,\left\lceil v \beta^{-\frac{1}{3}}\right\rceil\right),
$$

where we use that $A_{\beta}=e^{e_{\beta}} P$ with $e_{\beta}\left(\left\lceil u \beta^{-\frac{1}{3}}\right\rceil,\left\lceil v \beta^{-\frac{1}{3}}\right\rceil\right) \geq \Leftrightarrow 9 N^{2} \beta^{\frac{1}{3}}$ on the integration area (see (2.4)). Put $w=\beta^{-\frac{1}{6}}(v \Leftrightarrow u)$. Then by Lemma 3(i)

$$
\begin{aligned}
F_{\beta}^{2}\left(x_{\beta}\right) \leq & \Leftrightarrow \frac{1}{2} \beta^{-\frac{2}{3}} e^{-9 N^{2} \beta^{\frac{1}{3}}} \int_{\epsilon}^{N} d u \int_{-M}^{M} d w \beta^{\frac{1}{6}}\left[x_{\beta}(u) \Leftrightarrow x_{\beta}\left(u+w \beta^{\frac{1}{6}}\right)\right]^{2} \\
& \times\left\{\frac{1}{\sqrt{2 \pi 2 u \beta^{-\frac{1}{3}}}} \exp \left[\Leftrightarrow \frac{w^{2}}{4 u}\right]\right\}\left(1+o\left(\beta^{\frac{1}{9}}\right)\right),
\end{aligned}
$$

where the error term is uniform on the integration area. Collecting all the powers of $\beta$, we get the claim.

To investigate the limit of the integral in (2.14) as $\beta \downarrow 0$, we proceed with a technical fact contained in Steps 2 and 3 below. Let $T_{h}$ be the translation operator defined by $T_{h} x_{\beta}(\cdot)=$ $x_{\beta}(\cdot+h)$.

STEP 2 For every $0<a<b<\infty$

$$
\liminf _{h \rightarrow 0, \beta \downarrow 0} \int_{a}^{b}\left\{\frac{1}{h}\left[T_{h} x_{\beta} \Leftrightarrow x_{\beta}\right](u)\right\}^{2} \geq \int_{a}^{b}\left[x^{\prime}(u)\right]^{2} d u .
$$

Proof. Since (2.17) is trivial when the liminf is infinite, we may assume that the liminf is finite, say $L$. Pick any subsequence $h_{n}, \beta_{n}$ along which the liminf is reached, and put $y_{n}=\frac{1}{h_{n}}\left[T_{h_{n}} x_{\beta_{n}} \Leftrightarrow x_{\beta_{n}}\right]$. Then, because $\left\|y_{n}\right\|_{L^{2}[a, b]} \leq L+1<\infty$ for $n$ large enough, it follows from the Banach-Alaoglu theorem (Rudin (1991) Theorem 3.15) that there exists a subsequence $\left(y_{n_{k}}\right)$ and a $y \in L^{2}[a, b]$ such that

$$
y_{n_{k}} \rightarrow y \text { weakly in } L^{2}[a, b](k \rightarrow \infty) .
$$

Thus, for any $\phi \in C_{c}^{1}(a, b)=\left\{\phi \in C^{1}(a, b): \operatorname{supp}(\phi) \subset(a, b)\right\}$

$$
\int_{a}^{b} y_{n_{k}}(u) \phi(u) d u \rightarrow \int_{a}^{b} y(u) \phi(u) d u \quad(k \rightarrow \infty) .
$$

Next, the 1.h.s. of (2.19) can be rewritten as

$$
\begin{aligned}
\int_{a}^{b} y_{n_{k}}(u) \phi(u) d u & =\int_{a}^{b} \frac{1}{h_{n}}\left[T_{h_{n}} x_{\beta_{n}} \Leftrightarrow x_{\beta_{n}}\right](u) \phi(u) d u \\
& =\int_{a+h_{n} 1_{\left\{h_{n}<0\right\}}^{b+h_{n}} 1_{\left\{h_{n}>0\right\}}} x_{\beta_{n}}(u) \frac{1}{h_{n}}\left[T_{-h_{n}} \phi \Leftrightarrow \phi\right](u) d u \\
& =\int_{a}^{b} x_{\beta_{n}}(u) \frac{1}{h_{n}}\left[T_{-h_{n}} \phi \Leftrightarrow \phi\right](u) d u+o(1) \quad(n \rightarrow \infty) .
\end{aligned}
$$

The last equality holds because $\left\|x_{\beta_{n}}\right\|_{L^{2}\left(\mathbb{R}^{+}\right)}=1$ and $\left|\frac{1}{h_{n}}\left[T_{-h_{n}} \phi \Leftrightarrow \phi\right]\right| \leq \max _{u \in \mathbb{R}^{+}}\left|\phi^{\prime}(u)\right|<\infty$. Let $n \rightarrow \infty$ and note that by the latter property

$$
\frac{1}{h_{n}}\left[T_{-h_{n}} \phi \Leftrightarrow \phi\right] \rightarrow \Leftrightarrow \phi^{\prime} \text { pointwise and weakly in } L^{2}[a, b]
$$


Together with $x_{\beta_{n}} \rightarrow^{L^{2}} x,(2.21)$ implies that the last integral in $(2.20)$ tends to $\int_{a}^{b} x(u)\left[\Leftrightarrow \phi^{\prime}(u)\right] d u=$ $\int_{a}^{b} x^{\prime}(u) \phi(u) d u$ (recall from (1.13) that $x \in Y \subset C^{1}\left(\mathbb{R}_{0}^{+}\right)$). Since $C_{c}^{1}(a, b)$ is dense in $L^{2}[a, b]$ in the weak topology, we thus have from (2.19)

$$
y=x^{\prime} \text { a.e. on }[a, b] \text {. }
$$

The claim now follows by combining (2.18) and (2.22), and noting that $\|\cdot\|_{L^{2}[a, b]}$ is lower semicontinuous in the weak topology: $L=\lim _{k \rightarrow \infty}\left\|y_{n_{k}}\right\|_{L^{2}[a, b]} \geq\|y\|_{L^{2}[a, b]}=\left\|x^{\prime}\right\|_{L^{2}[a, b]}$.

STEP 3 For every $\epsilon>0$ and $N$ finite, every $f: \mathbb{R}^{+} \rightarrow \mathbb{R}^{+}$bounded and continuous, and every $w \in \mathbb{R}$

$$
\liminf _{\beta \backslash 0} \int_{\epsilon}^{N} d u f(u)\left[\frac{1}{\beta^{\frac{1}{6}}}\left\{x_{\beta}(u) \Leftrightarrow x_{\beta}\left(u+w \beta^{\frac{1}{6}}\right)\right\}\right]^{2} \geq \int_{\epsilon}^{N} d u f(u)\left[w x^{\prime}(u)\right]^{2} .
$$

Proof. Pick any sequence $\left(f_{n}\right)$ of functions on $\mathbb{R}^{+}$such that

(i) $f_{n}(u)=f_{n, k}$ for $c_{n, k-1}<u \leq c_{n, k}\left(k=1, \ldots, n ; c_{n, 0}=\epsilon, c_{n, n}=N\right)$

(ii) $f_{n} \leq f$

(iii) $f_{n} \uparrow f$ in sup-norm on $[\epsilon, N]$ as $n \rightarrow \infty$.

Then, by (i) and (ii),

$$
\begin{aligned}
\text { l.h.s. (2.23) } & \geq \liminf _{\beta \downarrow 0} \int_{\epsilon}^{N} d u f_{n}(u)\left[\frac{1}{\beta^{\frac{1}{6}}}\left\{x_{\beta}(u) \Leftrightarrow x_{\beta}\left(u+w \beta^{\frac{1}{6}}\right)\right\}\right]^{2} \\
& \geq \sum_{k=1}^{n} f_{n, k} \liminf \operatorname{in}_{\beta \downarrow 0} \int_{c_{n, k-1}}^{c_{n, k}} d u\left[\frac{1}{\beta^{\frac{1}{6}}}\left\{x_{\beta}(u) \Leftrightarrow x_{\beta}\left(u+w \beta^{\frac{1}{6}}\right)\right\}\right]^{2} \\
& \geq \sum_{k=1}^{n} f_{n, k} \int_{c_{n, k-1}}^{c_{n, k}} d u\left[w x^{\prime}(u)\right]^{2} \\
& =\int_{\epsilon}^{N} d u f_{n}(u)\left[w x^{\prime}(u)\right]^{2},
\end{aligned}
$$

where in the third inequality we use (2.17) with $h=w \beta^{\frac{1}{6}}$ and $a=c_{n, k-1}, b=c_{n, k}$ ( $k=$ $1, \ldots, n)$. Now let $n \rightarrow \infty$ and use (iii) together with Fatou to get the claim in (2.23).

Using (2.23) we can now finish the proof of Lemma 7 . Indeed, continuing with (2.14), we get

$$
\begin{aligned}
\limsup _{\beta \downarrow 0} F_{\beta}^{2}\left(x_{\beta}\right) & \leq \Leftrightarrow \frac{1}{2} \int_{-M}^{M} d w \int_{\epsilon}^{N} d u N_{2 u}(w)\left[w x^{\prime}(u)\right]^{2} \\
& =\Leftrightarrow \frac{1}{2} \int_{\epsilon}^{N} d u\left[x^{\prime}(u)\right]^{2} \int_{-M}^{M} d w w^{2} N_{2 u}(w) .
\end{aligned}
$$

Finally, let $M \rightarrow \infty$ and note that $\int_{-\infty}^{\infty} d w w^{2} N_{2 u}(w)=2 u$. Then let $N \rightarrow \infty$ and $\epsilon \downarrow 0$ to get the claim in Lemma 7 .

Lemma $8 \forall x \in Y$ such that $\int_{0}^{\infty} u^{2} x^{2}(u) d u<\infty: \liminf _{\beta \downarrow 0} F_{\beta}^{2}(x) \geq F^{2}(x)$.

Proof. The double integral defining $F_{\beta}^{2}(x)$ is split into three parts, which we estimate separately in Steps 1-3 below. 


\section{STEP 1}

$$
\lim _{\beta \downarrow 0} \Leftrightarrow \frac{1}{2} \beta^{-\frac{2}{3}} \int_{0}^{\infty} d u \int_{0}^{\infty} d v 1_{\left\{u>\beta^{-\frac{1}{6}} \text { or } v>\beta^{\left.-\frac{1}{6}\right\}}\right.}[x(u) \Leftrightarrow x(v)]^{2} A_{\beta}\left(\left\lceil u \beta^{-\frac{1}{3}}\right\rceil,\left\lceil v \beta^{-\frac{1}{3}}\right\rceil\right)=0 .
$$

Proof. First consider the part where $u>\beta^{-\frac{1}{6}}, v \geq 0$. By (2.5)(ii) and Lemma 3(ii)

$$
\begin{aligned}
& \Leftrightarrow \frac{1}{2} \beta^{-\frac{2}{3}} \int_{\beta^{-\frac{1}{6}}}^{\infty} d u \int_{0}^{\infty} d v[x(u) \Leftrightarrow x(v)]^{2} A_{\beta}\left(\left\lceil u \beta^{-\frac{1}{3}}\right\rceil,\left\lceil v \beta^{-\frac{1}{3}}\right\rceil\right) \\
& \geq \Leftrightarrow \frac{1}{2} \beta^{-\frac{2}{3}} e^{\frac{1}{4} a^{2} \beta^{\frac{1}{3}}}\left\{\int_{\beta^{-\frac{1}{6}}}^{\infty} d u \int_{\frac{1}{2} \beta^{-\frac{1}{6}}}^{\infty} d v[x(u) \Leftrightarrow x(v)]^{2} P\left(\left\lceil u \beta^{-\frac{1}{3}}\right\rceil,\left\lceil v \beta^{-\frac{1}{3}}\right\rceil\right)\right. \\
&\left.+\int_{\beta^{-\frac{1}{6}}}^{\infty} d u \int_{0}^{\frac{1}{2} \beta^{-\frac{1}{6}}} d v[x(u) \Leftrightarrow x(v)]^{2} e^{-c_{1} \beta^{-\frac{1}{3}} \frac{(u-v)^{2}}{(u+v)}}\right\} \\
&= \Leftrightarrow \frac{1}{2} \beta^{-\frac{2}{3}} e^{\frac{1}{4} a^{2} \beta^{\frac{1}{3}}}\left\{\int_{\beta^{-\frac{1}{6}}}^{\infty} d u \int_{\frac{1}{2} \beta^{-\frac{1}{6}}}^{\infty} d v[x(u) \Leftrightarrow x(v)]^{2} P\left(\left\lceil u \beta^{-\frac{1}{3}}\right\rceil,\left\lceil v \beta^{-\frac{1}{3}}\right\rceil\right)\right. \\
&\left.+\mathcal{O}\left(e^{-\frac{1}{12} c_{1} \beta^{-\frac{1}{2}}}\right)\right\},
\end{aligned}
$$

where $c_{1}$ is the constant in Lemma 3 (ii). To get the error term we have used that ( $u \Leftrightarrow$ $v)^{2} /(u+v) \geq \frac{1}{3}(u \Leftrightarrow v)$ on the integration area. The double integral in the r.h.s. of (2.28) can be bounded above by

$$
\begin{aligned}
& \int_{\frac{1}{2} \beta^{-\frac{1}{6}}}^{\infty} d u \int_{\frac{1}{2} \beta^{-\frac{1}{6}}}^{\infty} d v[x(u) \Leftrightarrow x(v)]^{2} P\left(\left\lceil u \beta^{-\frac{1}{3}}\right\rceil,\left\lceil v \beta^{-\frac{1}{3}}\right\rceil\right) \\
& \quad \leq 2 \int_{\frac{1}{2} \beta^{-\frac{1}{6}}}^{\infty} d u x^{2}(u) \int_{\frac{1}{2} \beta^{-\frac{1}{6}}}^{\infty} d v P\left(\left\lceil u \beta^{-\frac{1}{3}}\right\rceil,\left\lceil v \beta^{-\frac{1}{3}}\right\rceil\right) \\
& \quad \leq 2 \beta^{\frac{1}{3}} \int_{\frac{1}{2} \beta^{-\frac{1}{6}}}^{\infty} d u x^{2}(u) .
\end{aligned}
$$

Hence

$$
\begin{aligned}
\text { r.h.s. }(2.28) & \geq \Leftrightarrow\left[1+\mathcal{O}\left(\beta^{\frac{1}{3}}\right)\right] \beta^{-\frac{1}{3}} \int_{\frac{1}{2} \beta^{-\frac{1}{6}}}^{\infty} d u x^{2}(u) \\
& \geq \Leftrightarrow 4\left[1+\mathcal{O}\left(\beta^{\frac{1}{3}}\right)\right] \int_{\frac{1}{2} \beta^{-\frac{1}{6}}}^{\infty} d u u^{2} x^{2}(u) \\
& =o(1) .
\end{aligned}
$$

By symmetry, the same estimate holds for the part with $u \geq 0, v>\beta^{-\frac{1}{6}}$.

\section{STEP 2}

$$
\lim _{\beta \downarrow 0} \Leftrightarrow \frac{1}{2} \beta^{-\frac{2}{3}} \int_{0}^{\infty} d u \int_{0}^{\infty} d v 1_{\left\{u, v \leq \beta^{-\frac{1}{6}},|u-v|>\beta^{\frac{1}{24}}\right\}}[x(u) \Leftrightarrow x(v)]^{2} A_{\beta}\left(\left\lceil u \beta^{-\frac{1}{3}}\right\rceil,\left\lceil v \beta^{-\frac{1}{3}}\right\rceil\right)=0 .
$$

Proof. By (2.5)(ii) and Lemma 3(ii), the integral in the 1.h.s. of (2.31) can be bounded below by

$$
\begin{aligned}
\Leftrightarrow \frac{1}{2} \beta^{-\frac{2}{3}} & e^{\frac{1}{4} a^{2} \beta^{\frac{1}{3}}} \int_{0}^{\beta^{-\frac{1}{6}}} d u \int_{0}^{\beta^{-\frac{1}{6}}} d v 1_{\left\{|u-v|>\beta^{\frac{1}{24}}\right\}}[x(u) \Leftrightarrow x(v)]^{2} e^{-c_{1} \beta^{-\frac{1}{3}} \frac{(u-v)^{2}}{(u+v)}} \\
& \geq \Leftrightarrow \frac{1}{2} \beta^{-\frac{2}{3}} e^{\frac{1}{4} a^{2} \beta^{\frac{1}{3}}} e^{-\frac{1}{2} c_{1} \beta^{-\frac{1}{12}}} \int_{0}^{\beta^{-\frac{1}{6}}} d u \int_{0}^{\beta^{-\frac{1}{6}}} d v\left[x^{2}(u)+x^{2}(v)\right] \\
& =\mathcal{O}\left(e^{-\frac{1}{4} c_{1} \beta^{-\frac{1}{12}}}\right)
\end{aligned}
$$


where $c_{1}$ is the constant in Lemma 3(ii).

STEP 3

$$
\begin{aligned}
& \liminf _{\beta \downarrow 0} \Leftrightarrow \frac{1}{2} \beta^{-\frac{2}{3}} \int_{0}^{\beta^{-\frac{1}{6}}} d u \int_{0}^{\beta^{-\frac{1}{6}}} d v 1_{\left\{|u-v| \leq \beta \frac{1}{24}\right\}}[x(u) \Leftrightarrow x(v)]^{2} A_{\beta}\left(\left\lceil u \beta^{-\frac{1}{3}}\right\rceil,\left\lceil v \beta^{-\frac{1}{3}}\right\rceil\right) \\
& \quad=\liminf _{\beta \downarrow 0} F_{\beta}^{2}(x) \\
& \geq F^{2}(x) .
\end{aligned}
$$

Proof. By (2.5)(ii) and the mean value theorem we have

$$
\begin{aligned}
& \Leftrightarrow \frac{1}{2} \beta^{-\frac{2}{3}} \int_{0}^{\beta^{-\frac{1}{6}}} d u \int_{0}^{\beta^{-\frac{1}{6}}} d v 1_{\left\{|u-v| \leq \beta^{\frac{1}{24}}\right\}}[x(u) \Leftrightarrow x(v)]^{2} A_{\beta}\left(\left\lceil u \beta^{-\frac{1}{3}}\right\rceil,\left\lceil v \beta^{-\frac{1}{3}}\right\rceil\right) \\
& \quad \geq \Leftrightarrow \frac{1}{2} \beta^{-\frac{2}{3}} e^{\frac{1}{4} a^{2} \beta^{\frac{1}{3}}} \int_{0}^{\infty} d u \int_{0}^{\infty} d v 1_{\left\{|u-v| \leq \beta \frac{1}{24}\right\}}\left[(u \Leftrightarrow v) x^{\prime}\left(\xi_{u v}\right)\right]^{2} P\left(\left\lceil u \beta^{-\frac{1}{3}}\right\rceil,\left\lceil v \beta^{-\frac{1}{3}}\right\rceil\right)
\end{aligned}
$$

for some $\xi_{u v}$ between $u$ and $v$. Let, as in (2.34),

$$
I_{\beta}(u)=\beta^{-\frac{2}{3}} \int_{0}^{\infty} d v 1_{\left\{|u-v| \leq \beta^{\frac{1}{24}}\right\}}(u \Leftrightarrow v)^{2} P\left(\left\lceil u \beta^{-\frac{1}{3}}\right\rceil,\left\lceil v \beta^{-\frac{1}{3}}\right\rceil\right) .
$$

Then, because $x \in C^{1}\left(\mathbb{R}_{0}^{+}\right)$, it follows that

$$
\text { r.h.s. }(2.34)=\Leftrightarrow \frac{1}{2} e^{\frac{1}{4} a^{2} \beta^{\frac{1}{3}}} \int_{0}^{\infty} d u I_{\beta}(u)\left[x^{\prime}\left(\xi_{u}\right)\right]^{2}
$$

for some $\xi_{u} \in\left[u \Leftrightarrow \beta^{\frac{1}{24}}, u+\beta^{\frac{1}{24}}\right] \cap \mathbb{R}_{0}^{+}$.

Next, using (1.17) we can estimate

$$
\begin{aligned}
I_{\beta}(u) & \leq \beta^{-\frac{2}{3}} \int_{0}^{\infty} d v(u \Leftrightarrow v)^{2} P\left(\left\lceil u \beta^{-\frac{1}{3}}\right\rceil,\left\lceil v \beta^{-\frac{1}{3}}\right\rceil\right) \\
& =\beta^{\frac{1}{3}} \sum_{j \geq 1} P\left(\left\lceil u \beta^{-\frac{1}{3}}\right\rceil, j\right)\left\{\left(u \beta^{-\frac{1}{3}}\right)^{2} \Leftrightarrow\left(u \beta^{-\frac{1}{3}}\right)(2 j \Leftrightarrow 1)+\left(j^{2} \Leftrightarrow j+\frac{1}{3}\right)\right\} \\
& =\beta^{\frac{1}{3}}\left\{\left(u \beta^{-\frac{1}{3}}\right)^{2} \Leftrightarrow\left(u \beta^{-\frac{1}{3}}\right)\left(2\left\lceil u \beta^{-\frac{1}{3}}\right\rceil+1\right)+\left(\left\lceil u \beta^{-\frac{1}{3}}\right\rceil^{2}+3\left\lceil u \beta^{-\frac{1}{3}}\right\rceil+\frac{1}{3}\right)\right\} \\
& \leq 2 u+\frac{13}{3} \beta^{\frac{1}{3}} .
\end{aligned}
$$

Combining (2.34) and (2.36-37) with the estimates obtained in Steps 1 and 2, we now have

$$
F_{\beta}^{2}(x)=\text { l.h.s. }(2.34)+o(1) \geq \Leftrightarrow\left(1+\mathcal{O}\left(\beta^{\frac{1}{3}}\right)\right) \int_{0}^{\infty} d u\left(u+\frac{13}{6} \beta^{\frac{1}{3}}\right)\left[x^{\prime}\left(\xi_{u}\right)\right]^{2}+o(1) .
$$

Next, pick $\delta>0$ and define

$$
z_{k}^{\delta}=\sup _{(k-1) \delta<u \leq k \delta} u\left[x^{\prime}(u)\right]^{2} \quad(k \geq 1)
$$


Since $\xi_{u} \in\left[u \Leftrightarrow \beta^{\frac{1}{24}}, u+\beta^{\frac{1}{24}}\right] \cap \mathbb{R}_{0}^{+}$, it follows that for $\beta$ small enough

$$
\begin{aligned}
\int_{0}^{\infty} d u & \left(u+\frac{13}{6} \beta^{\frac{1}{3}}\right)\left[x^{\prime}\left(\xi_{u}\right)\right]^{2} \\
& \leq(1+\delta) \int_{\delta}^{\infty} d u \xi_{u}\left[x^{\prime}\left(\xi_{u}\right)\right]^{2}+2 \delta \int_{0}^{\delta} d u\left[x^{\prime}\left(\xi_{u}\right)\right]^{2} \\
& \leq(1+\delta) \sum_{k \geq 1}\left(\delta z_{k}^{\delta}+2 \beta^{\frac{1}{24}} \max \left\{z_{k}^{\delta}, z_{k+1}^{\delta}\right\}\right)+2 \delta \int_{0}^{\delta} d u \sup _{v \leq 2 \delta}\left[x^{\prime}(v)\right]^{2} \\
& \leq(1+\delta)\left(1+4 \delta^{-1} \beta^{\frac{1}{24}}\right) \sum_{k \geq 1} \delta z_{k}^{\delta}+2 \delta^{2} \sup _{v \leq 2 \delta}\left[x^{\prime}(v)\right]^{2} .
\end{aligned}
$$

Now let $\beta \downarrow 0$ followed by $\delta \downarrow 0$. Because $x \in C^{1}\left(\mathbb{R}_{0}^{+}\right)$, we have

$$
\begin{aligned}
\lim _{\delta \downarrow 0} \sum_{k \geq 1} \delta z_{k}^{\delta} & =\int_{0}^{\infty} u\left[x^{\prime}(u)\right]^{2} d u=\Leftrightarrow F^{2}(x) \\
\lim _{\delta \downarrow 0} \sup _{v \leq 2 \delta}\left[x^{\prime}(v)\right]^{2} & =\left[x^{\prime}(0+)\right]^{2}<\infty
\end{aligned}
$$

and so

$$
\liminf _{\beta \downarrow 0} \text { r.h.s. }(2.38) \geq F^{2}(x)
$$

Lemmas 5-8 show that $F_{\beta}$ epi-converges to $F$ on $Y$. To see why, recall (2.1) and note that if $\int_{0}^{\infty} u^{2} x^{2}(u) d u=\infty$, then $F(x) \leq F^{1}(x)=\Leftrightarrow \infty$. This proves Assumption (1) in Proposition 2 as was claimed in Proposition 3.

\section{An approximate maximizer of $F_{\beta}^{a}$}

Again we fix $a \in \mathbb{R}$ and suppress it from the notation. Like section 2, this section is technically somewhat involved, as it consists of a chain of estimates and inequalities that are needed to handle the approximation.

Define the scaled form of the eigenvector $\tau_{\beta}$ of $A_{\beta}$ as

$$
\bar{\tau}_{\beta}(u)=\beta^{-\frac{1}{6}} \tau_{\beta}(i) \text { for }(i \Leftrightarrow 1) \beta^{\frac{1}{3}}<u \leq i \beta^{\frac{1}{3}} \quad(i \geq 1) .
$$

By Lemma $1, \bar{\tau}_{\beta}$ is the unique maximizer of $F_{\beta}$. However, $\bar{\tau}_{\beta}$ is a step function and therefore $F\left(\bar{\tau}_{\beta}\right)$ is not defined, i.e., $\bar{\tau}_{\beta} \notin \bar{K}=\{x \in X: F(x) \geq \Leftrightarrow C\}$ (recall (1.13)). Thus, to apply Proposition 2, we must find an approximation of $\bar{\tau}_{\beta}$ that lies in $\bar{K}$ and approximates $F_{\beta}\left(\bar{\tau}_{\beta}\right)$ (i.e., we must prove Assumption 3(iii) in Proposition 2).

Proposition $4 \exists\left(\tilde{\tau}_{\beta}\right) \subset \bar{K}$ such that as $\beta \downarrow 0$

$$
\begin{aligned}
& \text { (i) }\left\|\bar{\tau}_{\beta} \Leftrightarrow \tilde{\tau}_{\beta}\right\|_{L^{2}} \rightarrow 0 \\
& \text { (ii) } 0 \leq F_{\beta}\left(\bar{\tau}_{\beta}\right) \Leftrightarrow F_{\beta}\left(\tilde{\tau}_{\beta}\right) \rightarrow 0 .
\end{aligned}
$$

The proof of Proposition 4 is contained in Lemmas 9-13 below. We shall see that it suffices to pick for $\tilde{\tau}_{\beta}$ the following linear and renormed interpolation of $\bar{\tau}_{\beta}$ :

$$
\begin{aligned}
& \tilde{\tau}_{\beta}=\hat{\tau}_{\beta}\left\|\hat{\tau}_{\beta}\right\|_{L^{2}}^{-1} \\
& \hat{\tau}_{\beta}(u)=\beta^{-\frac{1}{6}}\left\{\tau_{\beta}(i)+\left(u \beta^{-\frac{1}{3}} \Leftrightarrow i\right)\left(\tau_{\beta}(i) \Leftrightarrow \tau_{\beta}(i \Leftrightarrow 1)\right)\right\} \text { for }(i \Leftrightarrow 1) \beta^{\frac{1}{3}}<u \leq i \beta^{\frac{1}{3}} \quad(i \geq 1) .
\end{aligned}
$$


$\left(\right.$ put $\left.\tau_{\beta}(0)=\tau_{\beta}(1)\right)$.

We begin with two lemmas showing what is needed about $\tau_{\beta}$ in order to prove Proposition 4. Abbreviate $\Delta \tau_{\beta}(i)=\tau_{\beta}(i) \Leftrightarrow \tau_{\beta}(i \Leftrightarrow 1)(i \geq 1)$.

Lemma 9 (i) $\left\|\bar{\tau}_{\beta} \Leftrightarrow \tilde{\tau}_{\beta}\right\|_{L^{2}} \leq\left\|\Delta \tau_{\beta}\right\|_{l^{2}}+\tau_{\beta}^{2}(0)$.

(ii) $0 \leq F_{\beta}\left(\bar{\tau}_{\beta}\right) \Leftrightarrow F_{\beta}\left(\tilde{\tau}_{\beta}\right) \leq \lambda(\beta) \beta^{-\frac{1}{3}}\left\|\Delta \tau_{\beta}\right\|_{l^{2}}^{2}\left[1 \Leftrightarrow \frac{1}{6}\left\|\Delta \tau_{\beta}\right\|_{l^{2}}^{2}+\frac{1}{2} \tau_{\beta}^{2}(0)\right]^{-1}$.

Proof. (i) From (3.1) and (3.3) we compute

$$
\begin{aligned}
\left\|\bar{\tau}_{\beta} \Leftrightarrow \hat{\tau}_{\beta}\right\|_{L^{2}}^{2} & =\frac{1}{3}\left\|\Delta \tau_{\beta}\right\|_{l^{2}}^{2} \\
\left\|\hat{\tau}_{\beta}\right\|_{L^{2}}^{2} & =\left\|\tau_{\beta}\right\|_{l^{2}}^{2} \Leftrightarrow\left\langle\tau_{\beta}, \Delta \tau_{\beta}\right\rangle_{l^{2}}+\frac{1}{3}\left\|\Delta \tau_{\beta}\right\|_{l^{2}}^{2} .
\end{aligned}
$$

Using the relation $\left\langle\tau_{\beta}, \Delta \tau_{\beta}\right\rangle_{l^{2}}=\frac{1}{2}\left\|\Delta \tau_{\beta}\right\|_{l^{2}}^{2} \Leftrightarrow \frac{1}{2} \tau_{\beta}^{2}(0)$, together with (3.4-5) and $\left\|\tau_{\beta}\right\|_{l^{2}}=1$, we get

$$
\begin{aligned}
\left\|\bar{\tau}_{\beta} \Leftrightarrow \tilde{\tau}_{\beta}\right\|_{L^{2}} & \leq\left\|\bar{\tau}_{\beta} \Leftrightarrow \hat{\tau}_{\beta}\right\|_{L^{2}}+\left\|\hat{\tau}_{\beta} \Leftrightarrow \tilde{\tau}_{\beta}\right\|_{L^{2}} \\
& =\left\|\bar{\tau}_{\beta} \Leftrightarrow \hat{\tau}_{\beta}\right\|_{L^{2}}+\left\|\hat{\tau}_{\beta}\right\|_{L^{2}} \Leftrightarrow 1 \mid \\
& =\left(\frac{1}{3}\left\|\Delta \tau_{\beta}\right\|_{l^{2}}^{2}\right)^{\frac{1}{2}}+\left|\left[1 \Leftrightarrow \frac{1}{6}\left\|\Delta \tau_{\beta}\right\|_{l^{2}}^{2}+\frac{1}{2} \tau_{\beta}^{2}(0)\right]^{\frac{1}{2}} \Leftrightarrow 1\right| \\
& \leq\left(\frac{1}{3}\right)^{\frac{1}{2}}\left\|\Delta \tau_{\beta}\right\|_{l^{2}}+\frac{1}{6}\left\|\Delta \tau_{\beta}\right\|_{l^{2}}^{2}+\frac{1}{2} \tau_{\beta}^{2}(0) \\
& \leq\left(\left(\frac{1}{3}\right)^{\frac{1}{2}}+\frac{1}{3}\right)\left\|\Delta \tau_{\beta}\right\|_{l^{2}}+\frac{1}{2} \tau_{\beta}^{2}(0)
\end{aligned}
$$

where we use that $\left\|\Delta \tau_{\beta}\right\|_{l^{2}} \leq 2, \tau_{\beta}^{2}(0) \leq 1$.

(ii) From the definition of $F_{\beta}$ in (1.2) we get, after substitution of (3.1) and (3.3),

$$
\begin{aligned}
& F_{\beta}\left(\bar{\tau}_{\beta}\right)=\beta^{-\frac{1}{3}}\left\langle\tau_{\beta}, A_{\beta} \tau_{\beta}\right\rangle_{l^{2}} \Leftrightarrow \beta^{-\frac{1}{3}}\left\|\tau_{\beta}\right\|_{l^{2}}^{2} \\
& F_{\beta}\left(\hat{\tau}_{\beta}\right)=\beta^{-\frac{1}{3}}\left\langle\left(\tau_{\beta} \Leftrightarrow \frac{1}{2} \Delta \tau_{\beta}\right), A_{\beta}\left(\tau_{\beta} \Leftrightarrow \frac{1}{2} \Delta \tau_{\beta}\right)\right\rangle_{l^{2}} \Leftrightarrow \beta^{-\frac{1}{3}}\left\|\hat{\tau}_{\beta}\right\|_{L^{2}}^{2} .
\end{aligned}
$$

It follows from (3.7) that

$$
\begin{aligned}
F_{\beta}\left(\bar{\tau}_{\beta}\right) \Leftrightarrow F_{\beta}\left(\tilde{\tau}_{\beta}\right) & =F_{\beta}\left(\bar{\tau}_{\beta}\right) \Leftrightarrow \frac{1}{\left\|\hat{\tau}_{\beta}\right\|_{L^{2}}^{2}} F_{\beta}\left(\hat{\tau}_{\beta}\right) \\
& =\beta^{-\frac{1}{3}} \frac{1}{\left\|\hat{\gamma}_{\beta}\right\|_{L^{2}}^{2}}\left\{\frac{1}{3} \lambda(\beta)\left\|\Delta \tau_{\beta}\right\|_{l^{2}}^{2} \Leftrightarrow \frac{1}{4}\left\langle\Delta \tau_{\beta}, A_{\beta} \Delta \tau_{\beta}\right\rangle_{l^{2}}\right\},
\end{aligned}
$$

where in the second equality we use the symmetry of $A_{\beta}$ and the relations $A_{\beta} \tau_{\beta}=\lambda(\beta) \tau_{\beta}$ and (3.5). Finally, observe that $\left|\left\langle\Delta \tau_{\beta}, A_{\beta} \Delta \tau_{\beta}\right\rangle_{l^{2}}\right| \leq\left\langle\left|\Delta \tau_{\beta}\right|, A_{\beta}\left|\Delta \tau_{\beta}\right|\right\rangle_{l^{2}} \leq \lambda(\beta)|| \Delta \tau_{\beta} \|_{l^{2}}^{2}$ to get the claim.

\section{Lemma 10}

$$
\begin{aligned}
F\left(\tilde{\tau}_{\beta}\right) \geq \Leftrightarrow 2 & \sqrt{5}|a|\left(\beta^{\frac{2}{3}} \sum_{i \geq 1} i^{2} \tau_{\beta}^{2}(i)\right)^{\frac{1}{2}}\left[1 \Leftrightarrow \frac{1}{6}\left\|\Delta \tau_{\beta}\right\|_{l^{2}}^{2}+\frac{1}{2} \tau_{\beta}^{2}(0)\right]^{-\frac{1}{2}} \\
+ & \left\{20 \beta^{\frac{2}{3}} \sum_{i \geq 1} i^{2} \tau_{\beta}^{2}(i)+\beta^{-\frac{1}{3}} \sum_{i \geq 1} i \Delta \tau_{\beta}^{2}(i)\right\}\left[1 \Leftrightarrow \frac{1}{6}\left\|\Delta \tau_{\beta}\right\|_{l^{2}}^{2}+\frac{1}{2} \tau_{\beta}^{2}(0)\right]^{-1} .
\end{aligned}
$$


Proof. According to (2.1) and (2.3)

$$
F\left(\hat{\tau}_{\beta}\right)=\int_{0}^{\infty} d u\left\{\left(2 a u \Leftrightarrow 4 u^{2}\right) \hat{\tau}_{\beta}^{2}(u) \Leftrightarrow u\left[\hat{\tau}_{\beta}^{\prime}(u)\right]^{2}\right\} .
$$

Use (3.3) to obtain the estimates

$$
\begin{aligned}
& \int_{0}^{\infty} d u u^{2} \hat{\tau}_{\beta}^{2}(u) \leq \beta^{\frac{2}{3}} \sum_{i \geq 1} i^{2} \max \left\{\tau_{\beta}^{2}(i), \tau_{\beta}^{2}(i \Leftrightarrow 1)\right\} \\
& \int_{0}^{\infty} d u u\left[\hat{\tau}_{\beta}^{\prime}(u)\right]^{2} \leq \beta^{-\frac{1}{3}} \sum_{i \geq 1} i \Delta \tau_{\beta}^{2}(i) .
\end{aligned}
$$

Since $\int_{0}^{\infty} d u u \hat{\tau}_{\beta}^{2}(u) \leq\left(\int_{0}^{\infty} d u u^{2} \hat{\tau}_{\beta}^{2}(u)\right)^{\frac{1}{2}}\left\|\hat{\tau}_{\beta}\right\|_{L^{2}}$, we get the claim because $F\left(\tilde{\tau}_{\beta}\right)=\frac{1}{\left\|\hat{\tau}_{\beta}\right\|_{L^{2}}^{2}}$ $F_{\beta}\left(\hat{\tau}_{\beta}\right)$.

Lemmas 9 and 10 set the stage for the proof of Proposition 4. Namely, we now see that it suffices to prove the following estimates:

Lemma 11 There exist $C_{1}, C_{2}, C_{3}, C_{4}$ such that for $\beta$ small enough

$$
\begin{aligned}
& \text { (i) } \sum_{i \geq 1} i^{2} \tau_{\beta}^{2}(i) \leq C_{1} \beta^{-\frac{2}{3}} \\
& \text { (ii) } \sum_{i \geq 1} i \Delta \tau_{\beta}^{2}(i) \leq C_{2} \beta^{\frac{1}{3}} \\
& \text { (iii) } \tau_{\beta}^{2}(0) \leq C_{3} \beta^{\frac{1}{3}} \log \frac{1}{\beta} \\
& \text { (iv) }\left\|\Delta \tau_{\beta}\right\|_{l^{2}}^{2} \leq C_{4} \beta^{\frac{2}{3}} \log \frac{1}{\beta} .
\end{aligned}
$$

Indeed, Lemmas 11(iii-iv) and 9(i-ii) imply (3.2), while Lemmas 11(i-ii) and Lemma 10 imply that $F\left(\tilde{\tau}_{\beta}\right) \geq \Leftrightarrow C$ for $\beta$ small enough and $C$ sufficiently large, which guarantees that $\tilde{\tau}_{\beta} \in$ $\bar{K}=\overline{K_{C}^{a}}$.

In the proof of Lemma 11 we shall make use of the following two additional lemmas, the proof of which is deferred to section 4 :

Lemma $12 \forall \beta>0: \Delta \tau_{\beta}(i)=\tau_{\beta}(i) \Leftrightarrow \tau_{\beta}(i \Leftrightarrow 1) \leq 0$ for all $i \geq \frac{a}{2} \beta^{-\frac{1}{3}}$.

Lemma 13

$$
\begin{aligned}
& \lim \sup _{\beta \downarrow 0} \beta^{-\frac{1}{3}}[\lambda(\beta) \Leftrightarrow 1] \leq \frac{1}{4} a^{2} \\
& \begin{aligned}
& \liminf \beta \downarrow 0 \\
& \beta^{-\frac{1}{3}}[\lambda(\beta) \Leftrightarrow 1] \geq \frac{1}{2 \pi} a^{2} \Leftrightarrow \frac{2}{a} \quad(a>1) \\
& \frac{1}{\pi} a \Leftrightarrow \frac{1}{2 \pi} \Leftrightarrow 2 \quad(a \leq 1) .
\end{aligned}
\end{aligned}
$$

Proof of Lemma 11(i).

STEP 1 For every $\epsilon>0$ small enough there exists $C_{5}$ such that

$$
\sum_{i \leq \epsilon \beta^{-\frac{1}{2}}} i^{2} \tau_{\beta}^{2}(i) \leq C_{5} \beta^{-\frac{2}{3}} \text { for } \beta \text { small enough. }
$$

Proof. We start with the trivial inequality

$$
\frac{1}{2} \sum_{i, j}\left[\tau_{\beta}(i) \Leftrightarrow \tau_{\beta}(j)\right]^{2} A_{\beta}(i, j) \geq 0 .
$$


The 1.h.s. of (3.15) can be written out and estimated from above as follows:

$$
\begin{aligned}
{[1 \Leftrightarrow \lambda(\beta)] } & +\sum_{i, j} \tau_{\beta}^{2}(i)\left[A_{\beta}(i, j) \Leftrightarrow P(i, j)\right] \\
\leq & {[1 \Leftrightarrow \lambda(\beta)]+\sum_{i \leq \epsilon \beta^{-\frac{1}{2}}} \tau_{\beta}^{2}(i) \sum_{j}\left[e^{e_{\beta}(i, j)} \Leftrightarrow 1\right] P(i, j) } \\
\leq & {[1 \Leftrightarrow \lambda(\beta)]+\sum_{i \leq \epsilon \beta^{-\frac{1}{2}}} \tau_{\beta}^{2}(i) \sum_{j}\left[e_{\beta}(i, j)+e_{\beta}^{2}(i, j)\right] P(i, j) . }
\end{aligned}
$$

For the two inequalities we refer to (2.4-5) (use that $e^{t} \leq 1+t+t^{2}$ for $t \ll 1$ and $t \leq 0$ ). The sum over $j$ has been evaluated in (2.8). Using that $i \leq \epsilon \beta^{-\frac{1}{2}}$, we get

r.h.s. (3.16)

$$
\leq[1 \Leftrightarrow \lambda(\beta)]+\beta^{\frac{1}{3}} \sum_{i \leq \epsilon \beta^{-\frac{1}{2}}} \tau_{\beta}^{2}(i)\left\{2 a\left(i \beta^{\frac{1}{3}}\right) \Leftrightarrow\left(4 \Leftrightarrow 168 \epsilon^{2} \Leftrightarrow 6 a \beta^{\frac{1}{3}}\right)\left(i \beta^{\frac{1}{3}}\right)^{2}\right\} .
$$

Combining (3.15-17) we arrive at the following inequality:

$$
\begin{aligned}
\left(4 \Leftrightarrow 168 \epsilon^{2}\right. & \left.\Leftrightarrow 6 a \beta^{\frac{1}{3}}\right) \beta^{\frac{2}{3}} \sum_{i \leq \epsilon \beta^{-\frac{1}{2}}} i^{2} \tau_{\beta}^{2}(i) \\
& \leq \beta^{-\frac{1}{3}}[1 \Leftrightarrow \lambda(\beta)]+2 a \beta^{\frac{1}{3}} \sum_{i \leq \epsilon \beta^{-\frac{1}{2}}} i \tau_{\beta}^{2}(i) .
\end{aligned}
$$

Now, $\beta^{-\frac{1}{3}}[1 \Leftrightarrow \lambda(\beta)] \leq C_{6}$ by Lemma 13 . Moreover, by Cauchy-Schwarz

$$
\sum_{i \leq \epsilon \beta^{-\frac{1}{2}}} i \tau_{\beta}^{2}(i) \leq\left(\sum_{i \leq \epsilon \beta^{-\frac{1}{2}}} i^{2} \tau_{\beta}^{2}(i)\right)^{\frac{1}{2}}
$$

Hence the claim in (3.14) follows for $\epsilon$ such that $168 \epsilon^{2}<4$.

STEP 2 For every $\epsilon>0$ small enough there exists $C_{7}$ such that

$$
\sum_{i>\in \beta^{-\frac{1}{2}}} \tau_{\beta}^{2}(i) \leq C_{7} \beta^{\frac{1}{3}} \text { for } \beta \text { small enough. }
$$

Proof. Rewrite (3.15) as (recall also (3.16))

$$
\sum_{i>\epsilon \beta^{-\frac{1}{2}}} \tau_{\beta}^{2}(i) \sum_{j}\left[1 \Leftrightarrow e^{e_{\beta}(i, j)}\right] P(i, j) \leq[1 \Leftrightarrow \lambda(\beta)]+\sum_{i \leq \epsilon \beta^{-\frac{1}{2}}} \tau_{\beta}^{2}(i) \sum_{j}\left[e^{e_{\beta}(i, j)} \Leftrightarrow 1\right] P(i, j) .
$$

Since $e_{\beta}(i, j) \leq \frac{1}{4} a^{2} \beta^{\frac{1}{3}}$ for $i, j \geq 1$ and $e_{\beta}(i, j) \leq \Leftrightarrow \frac{1}{2} \epsilon^{2}$ for $i>\epsilon \beta^{-\frac{1}{2}}, j \geq 1$ (see (2.4-5)), we get

$$
\left(1 \Leftrightarrow e^{-\frac{1}{2} \epsilon^{2}}\right) \sum_{i>\epsilon \beta^{-\frac{1}{2}}} \tau_{\beta}^{2}(i) \leq C_{6} \beta^{\frac{1}{3}}+\left(e^{\frac{1}{4} a^{2} \beta^{\frac{1}{3}}} \Leftrightarrow 1\right) .
$$

This implies the claim in (3.20).

STEP 3 For every $\epsilon>0$ there exists $C_{8}$ such that

$$
\sum_{i>\epsilon \beta^{-\frac{1}{2}}} i^{2} \tau_{\beta}^{2}(i) \leq C_{8} \beta^{-\frac{2}{3}} \text { for } \beta \text { small enough. }
$$


Proof. Pick $i>\epsilon \beta^{-\frac{1}{2}}$ and $\delta>0$ arbitrary. Then, using (2.4) and Lemma 3(ii), we see that there exists $C(\delta)>0$ such that

$$
\begin{aligned}
\tau_{\beta}(i) & =\frac{1}{\lambda(\beta)} \sum_{j} A_{\beta}(i, j) \tau_{\beta}(j) \\
& =\frac{1}{\lambda(\beta)} \sum_{j} e^{e_{\beta}(i, j)} P(i, j) \tau_{\beta}(j) \\
& \leq \frac{1}{\lambda(\beta)} e^{-\frac{1}{2} \epsilon^{2}} \sum_{j} P(i, j) \tau_{\beta}(j) \\
& \leq(1+\delta) e^{-\frac{1}{2} \epsilon^{2}} \sum_{j>(1-\delta) i} P(i, j) \tau_{\beta}(j)+\mathcal{O}\left(e^{-i C(\delta)}\right) .
\end{aligned}
$$

Using (3.24) we get

$$
\begin{aligned}
\sum_{i>\epsilon \beta^{-\frac{1}{2}}} i^{2} \tau_{\beta}^{2}(i) & \leq(1+\delta) e^{-\frac{1}{2} \epsilon^{2}} \sum_{i>\epsilon \beta^{-\frac{1}{2}}} i^{2} \sum_{j>(1-\delta) i} \tau_{\beta}(i) P(i, j) \tau_{\beta}(j)+o(1) \\
& \leq \frac{(1+\delta)}{(1-\delta)^{2}} e^{-\frac{1}{2} \epsilon^{2}} \sum_{i>\epsilon \beta^{-\frac{1}{2}}}\lceil(1 \Leftrightarrow \delta) i\rceil^{2} \tau_{\beta}^{2}(\lceil(1 \Leftrightarrow \delta) i\rceil)+o(1) \\
& \leq \frac{(1+\delta)}{(1-\delta)^{3}} e^{-\frac{1}{2} \epsilon^{2}} \sum_{i>(1-\delta) \epsilon \beta^{-\frac{1}{2}}} i^{2} \tau_{\beta}^{2}(i)+o(1) \\
& \leq \frac{(1+\delta)}{(1-\delta)^{3}} e^{-\frac{1}{2} \epsilon^{2}}\left\{\sum_{i>\epsilon \beta^{-\frac{1}{2}}} i^{2} \tau_{\beta}^{2}(i)+\epsilon^{2} \beta^{-1} C_{7} \beta^{\frac{1}{3}}\right\}+o(1)
\end{aligned}
$$

The second and the third inequality use Lemma 12, the fourth uses (3.20) with $\epsilon$ replaced by $(1 \Leftrightarrow \delta) \epsilon$. Now pick $\delta$ so small that $\frac{(1+\delta)}{(1-\delta)^{3}}<e^{\frac{1}{4} \epsilon^{2}}$. Then we obtain

$$
\left(1 \Leftrightarrow e^{-\frac{1}{4} \epsilon^{2}}\right) \sum_{i>\epsilon \beta^{-\frac{1}{2}}} i^{2} \tau_{\beta}^{2}(i) \leq e^{-\frac{1}{4} \epsilon^{2}} \epsilon^{2} C_{7} \beta^{-\frac{2}{3}}+o(1) .
$$

This proves the claim in $(3.23)$.

Steps 1-3 complete the proof of Lemma 11(i).

\section{Proof of Lemma 11(ii).}

STEP 4 For all $\beta$

$$
\sum_{i \geq 1} i \Delta \tau_{\beta}^{2}(i+1)=\frac{1}{\lambda(\beta)} \sum_{i, j \geq 1}(i+j \Leftrightarrow 1)\left[1 \Leftrightarrow e^{e_{\beta}(i+1, j)-e_{\beta}(i, j)}\right] \tau_{\beta}(i) A_{\beta}(i, j) \tau_{\beta}(j) .
$$

Proof. Write out

$$
\begin{aligned}
\sum_{i} i \Delta \tau_{\beta}^{2}(i+1) & =\sum_{i} i\left[\tau_{\beta}(i+1) \Leftrightarrow \tau_{\beta}(i)\right]^{2} \\
& =\sum_{i} i\left[\tau_{\beta}^{2}(i+1)+\tau_{\beta}^{2}(i)\right] \Leftrightarrow \frac{1}{\lambda(\beta)} \sum_{i, j} 2 i \tau_{\beta}(i) A_{\beta}(i+1, j) \tau_{\beta}(j) .
\end{aligned}
$$

Now substitute the relation (see $(0.10-11)$ )

$$
\begin{aligned}
A_{\beta}(i+1, j) & =e^{e_{\beta}(i+1, j)} P(i+1, j) \\
& =e^{e_{\beta}(i+1, j) \frac{i+j-1}{2 i}} P(i, j) \\
& =e^{e_{\beta}(i+1, j)-e_{\beta}(i, j)} \frac{i+j-1}{2 i} A_{\beta}(i, j) .
\end{aligned}
$$


This gives

$$
\begin{aligned}
\sum_{i} i \Delta \tau_{\beta}^{2}(i+1)= & \text { r.h.s. }(3.27)+\sum_{i} i\left[\tau_{\beta}^{2}(i+1)+\tau_{\beta}^{2}(i)\right] \\
& \Leftrightarrow \frac{1}{\lambda(\beta)} \sum_{i, j}(i+j \Leftrightarrow 1) \tau_{\beta}(i) A_{\beta}(i, j) \tau_{\beta}(j) .
\end{aligned}
$$

Both sums in the r.h.s. are equal to $\sum_{i}(2 i \Leftrightarrow 1) \tau_{\beta}^{2}(i)$ and therefore cancel out.

STEP 5 For $\beta$ small enough

$$
\frac{1}{\lambda(\beta)} \sum_{i, j \geq 1}(i+j \Leftrightarrow 1)\left[1 \Leftrightarrow e^{e_{\beta}(i+1, j)-e_{\beta}(i, j)}\right] \tau_{\beta}(i) A_{\beta}(i, j) \tau_{\beta}(j) \leq C_{9} \beta^{\frac{1}{3}} .
$$

Proof. By (2.4) we have $\boldsymbol{e}_{\beta}(i+1, j) \Leftrightarrow \boldsymbol{\epsilon}_{\beta}(i, j)=a \beta^{\frac{2}{3}} \Leftrightarrow \beta(2 i+2 j \Leftrightarrow 1)$. Hence

$$
\begin{aligned}
\text { l.h.s. (3.31) } & \leq \frac{1}{\lambda(\beta)} \sum_{i, j \geq 1}(i+j \Leftrightarrow 1)\left[e_{\beta}(i, j) \Leftrightarrow \boldsymbol{e}_{\beta}(i+1, j)\right] \tau_{\beta}(i) A_{\beta}(i, j) \tau_{\beta}(j) \\
& \leq \frac{1}{\lambda(\beta)} 2 \beta \sum_{i, j}(i+j)^{2} \tau_{\beta}(i) A_{\beta}(i, j) \tau_{\beta}(j) \\
& \leq 8 \beta \sum_{i} i^{2} \tau_{\beta}^{2}(i)
\end{aligned}
$$

(use that $e^{t} \geq 1+t$ for all $t$ ). In the third inequality we use the symmetry of $A_{\beta}$ and the fact that $\left\|A_{\beta}\right\|_{l^{2}}=\lambda(\beta)$. The claim now follows from Lemma 11(i).

Steps 4-5 complete the proof of Lemma 11(ii).

\section{Proof of Lemma 11(iii).}

STEP 6

$$
\tau_{\beta}^{2}(0) \leq C_{10} \beta^{\frac{1}{3}} \log \frac{1}{\beta} \text { for } \beta \text { small enough }
$$

Proof. By Cauchy-Schwarz, we have for every $N$

$$
\begin{aligned}
\tau_{\beta}(0) & =\tau_{\beta}(N) \Leftrightarrow \sum_{i=1}^{N} \Delta \tau_{\beta}(i) \\
& \leq \tau_{\beta}(N)+\left(\sum_{i=1}^{N} \frac{1}{i}\right)^{\frac{1}{2}}\left(\sum_{i=1}^{N} i \Delta \tau_{\beta}^{2}(i)\right)^{\frac{1}{2}} .
\end{aligned}
$$

Pick $N=\left\lceil\beta^{-\frac{1}{2}}\right\rceil$. Lemma 11(i) gives $\tau_{\beta}\left(\left\lceil\beta^{-\frac{1}{2}}\right\rceil\right) \leq C_{1} \beta^{\frac{1}{3}}$. Together with Lemma 11(ii) and the estimate $\sum_{i=1}^{\left\lceil\beta^{-\frac{1}{2}}\right\rceil} \frac{1}{i} \leq \log \frac{1}{\beta}$, the claim follows.

Step 6 completes the proof of Lemma 11(iii).

\section{Proof of Lemma 11(iv).}

STEP 7 For all $\beta$

$$
\begin{aligned}
\sum_{i \geq 1} \Delta \tau_{\beta}^{2}(i+1)= & \frac{2}{\lambda(\beta)} \sum_{\substack{(i, j) \neq(1, j) \\
i, j}}\left[1 \Leftrightarrow e^{e_{\beta}(i-1, j)-e_{\beta}(i, j)}\right] \tau_{\beta}(i) A_{\beta}(i, j) \tau_{\beta}(j) \\
& \Leftrightarrow \tau_{\beta}^{2}(1)\left[1 \Leftrightarrow \frac{2}{\lambda(\beta)} A_{\beta}(1,1)\right] .
\end{aligned}
$$


Proof. By Lemma 4 we have the following relation:

$$
A_{\beta}(i, j) \Leftrightarrow A_{\beta}(i \Leftrightarrow 1, j)=A_{\beta}(i, j \Leftrightarrow 1) \Leftrightarrow A_{\beta}(i, j)+2 A_{\beta}(i, j)\left[1 \Leftrightarrow e^{e_{\beta}(i-1, j)-e_{\beta}(i, j)}\right]
$$

(note that $e_{\beta}(i \Leftrightarrow 1, j)=e_{\beta}(i, j \Leftrightarrow 1)$ ). Hence

$$
\begin{aligned}
\sum_{i} \Delta \tau_{\beta}^{2}(i+1)= & \sum_{i}\left[\tau_{\beta}(i+1) \Leftrightarrow \tau_{\beta}(i)\right]^{2} \\
= & \tau_{\beta}^{2}(1)+2 \sum_{i \geq 2} \tau_{\beta}(i)\left[\tau_{\beta}(i) \Leftrightarrow \tau_{\beta}(i \Leftrightarrow 1)\right] \\
= & \tau_{\beta}^{2}(1)+\frac{2}{\lambda(\beta)} \sum_{i \geq 2} \sum_{j} \tau_{\beta}(i)\left[A_{\beta}(i, j) \Leftrightarrow A_{\beta}(i \Leftrightarrow 1, j)\right] \tau_{\beta}(j) \\
= & \tau_{\beta}^{2}(1)+\frac{2}{\lambda(\beta)} \sum_{i \geq 2} \sum_{j} \tau_{\beta}(i)\left[A_{\beta}(i, j \Leftrightarrow 1) \Leftrightarrow A_{\beta}(i, j)\right] \tau_{\beta}(j) \\
& +\frac{4}{\lambda(\beta)} \sum_{i \geq 2} \sum_{j}\left[1 \Leftrightarrow e^{e_{\beta}(i-1, j)-e_{\beta}(i, j)}\right] \tau_{\beta}(i) A_{\beta}(i, j) \tau_{\beta}(j) .
\end{aligned}
$$

The third term in the last expression is twice the sum in the r.h.s. of (3.35) except for the part with $i=1, j \geq 2$. The second term can be rewritten by carrying out the sum over $i$, namely (use that $A(i, 0)=0$ )

$$
\begin{aligned}
j=1: & \Leftrightarrow \frac{2}{\lambda(\beta)} \sum_{i \geq 2} \tau_{\beta}(i) A_{\beta}(i, 1) \tau_{\beta}(1) \\
& =\Leftrightarrow 2 \tau_{\beta}^{2}(1)+\frac{2}{\lambda(\beta)} \tau_{\beta}^{2}(1) A_{\beta}(1,1) \\
j \geq 2: & \frac{2}{\lambda(\beta)} \sum_{i \geq 2} \tau_{\beta}(i)\left[A_{\beta}(i, j \Leftrightarrow 1) \Leftrightarrow A_{\beta}(i, j)\right] \tau_{\beta}(j) \\
& =2 \tau_{\beta}(j)\left[\tau_{\beta}(j \Leftrightarrow 1) \Leftrightarrow \tau_{\beta}(j)\right] \Leftrightarrow \frac{2}{\lambda(\beta)} \tau_{\beta}(1)\left[A_{\beta}(1, j \Leftrightarrow 1) \Leftrightarrow A_{\beta}(1, j)\right] \tau_{\beta}(j) .
\end{aligned}
$$

Thus, after carrying out the sum over $j$, we see that (3.37) becomes

$$
\begin{aligned}
\sum_{i} \Delta \tau_{\beta}^{2}(i+1)= & \Leftrightarrow \sum_{j} \Delta \tau_{\beta}^{2}(j+1) \\
& +2\left\{\text { r.h.s. }(3.35) \Leftrightarrow \frac{2}{\lambda(\beta)} \sum_{j \geq 2}\left[1 \Leftrightarrow e^{e_{\beta}(0, j)-e_{\beta}(1, j)}\right] \tau_{\beta}(1) A_{\beta}(1, j) \tau_{\beta}(j)\right. \\
& \left.\quad+\tau_{\beta}^{2}(1)\left[1 \Leftrightarrow \frac{2}{\lambda(\beta)} A_{\beta}(1,1)\right]\right\} \\
& +\frac{2}{\lambda(\beta)} \tau_{\beta}^{2}(1) A_{\beta}(1,1) \Leftrightarrow \frac{2}{\lambda(\beta)} \sum_{j \geq 2} \tau_{\beta}(1)\left[A_{\beta}(1, j \Leftrightarrow 1) \Leftrightarrow A_{\beta}(1, j)\right] \tau_{\beta}(j) .
\end{aligned}
$$

Now, by (3.36) for $i=1$,

$$
2\left[1 \Leftrightarrow e^{e_{\beta}(0, j)-e_{\beta}(1, j)}\right] A_{\beta}(1, j)=\Leftrightarrow\left[A_{\beta}(1, j \Leftrightarrow 1) \Leftrightarrow A_{\beta}(1, j)\right]+A_{\beta}(1, j) .
$$

Hence (3.39) simplifies to

$$
\begin{aligned}
\sum_{i} \Delta \tau_{\beta}^{2}(i+1) & =\Leftrightarrow \sum_{j} \Delta \tau_{\beta}^{2}(j+1)+2 \text { r.h.s. (3.35) } \\
& +\left\{2 \tau_{\beta}^{2}(1) \Leftrightarrow \frac{2}{\lambda(\beta)} \tau_{\beta}^{2}(1) A_{\beta}(1,1) \Leftrightarrow \frac{2}{\lambda(\beta)} \sum_{j \geq 2} \tau_{\beta}(1) A_{\beta}(1, j) \tau_{\beta}(j)\right\} .
\end{aligned}
$$

But the term between braces is zero. 
STEP 8 For $\beta$ small enough

$$
\text { r.h.s. }(3.35) \leq C_{11} \beta^{\frac{2}{3}} \log \frac{1}{\beta} .
$$

Proof. The first term in (3.35) is easy to bound. Indeed, we have $e_{\beta}(i \Leftrightarrow 1, j) \Leftrightarrow e_{\beta}(i, j)=$ $\Leftrightarrow a \beta^{\frac{2}{3}}+\beta(2 i+2 j \Leftrightarrow 3)$ and hence we get

$$
\begin{aligned}
1^{\text {st }} \text { term in }(3.35) & \leq \frac{2}{\lambda(\beta)} \sum_{\substack{(i, j) \neq(1, j) \\
i, j}} a \beta^{\frac{2}{3}} \tau_{\beta}(i) A_{\beta}(i, j) \tau_{\beta}(j) \\
& \leq 2 a \beta^{\frac{2}{3}}
\end{aligned}
$$

(in the first inequality use that $e^{t} \geq 1+t$ for all $t$ ). For the second term in (3.35), use that $P(1,1)=\frac{1}{2}$ and $e_{\beta}(1,1)=a \beta^{\frac{2}{3}} \Leftrightarrow \beta$. Together with $\lambda(\beta) \geq 1 \Leftrightarrow C_{6} \beta^{\frac{1}{3}}$ (see below (3.18)) we get

$$
2^{\text {nd }} \text { term in }(3.35) \leq 2 \tau_{\beta}^{2}(1) C_{6} \beta^{\frac{1}{3}} \text { for } \beta \text { small enough. }
$$

Finally, use Step 6 to get the claim (recall that $\tau_{\beta}(0)=\tau_{\beta}(1)$ in $(3.3)$ ).

Steps 7-8 complete the proof of Lemma 11(iv).

Lemma 11 completes the proof of Proposition 4. Lemmas 12 and 13 will be proved in section 4 .

Proposition 4 shows that Assumption 3(iii) in Proposition 2 holds. We shall prove Assumptions $3(\mathrm{i})$,(ii) in section 5 .

\section{Proof of Lemmas 12 and 13}

\subsection{Proof of Lemma 12}

Let $\left(e_{i}\right)_{i \geq 1}$ be the canonical base of $l^{2}(\mathbb{N})$. Let $s=(s(i))_{i \geq 1}$ be any sequence of numbers in $(0, \infty)$ and let $t=(t(i))_{i \geq 1}$ be given by $t(1)=1, t(i)=\prod_{k=1}^{i-1} s(k)(i \geq 2)$. Define

$$
\begin{aligned}
& B_{s}=\left\{x \in l^{2}(\mathbb{N}): x \geq 0, x(i+1) \leq s(i) x(i)\right\} \\
& B_{s}^{0}=\left\{x=\sum_{j} c_{j} f_{j}: c_{j} \geq 0, c_{j} \neq 0 \text { finitely often }\right\}
\end{aligned}
$$

where $f_{j} \in l^{2}(\mathbb{N})$ is defined by

$$
f_{j}=\sum_{i=1}^{j} t(i) e_{i} .
$$

Lemma 14 (i) $B_{s}$ is a closed convex cone.

(ii) $B_{s}$ is the closure of $B_{s}^{0}$.

Proof. Elementary.

Recall footnote 6 . Since, for every $\beta>0, A_{\beta}$ is a continuous operator on $l^{2}(\mathbb{N})$, we have from Lemma 14(ii) that

$$
A_{\beta} B_{s} \subset B_{s} \Leftrightarrow A_{\beta} f_{j} \in B_{s} \text { for all } j \geq 1 \text {. }
$$


Since, for every $\beta>0, A_{\beta}$ is symmetric and has a spectral gap, we also know that $\lambda(\beta)^{-n} A_{\beta}^{n} x \rightarrow l^{2}$ $\left\langle x, \tau_{\beta}\right\rangle_{l^{2}} \tau_{\beta}(n \rightarrow \infty)$ for any $x \in l^{2}(\mathbb{N})$. Pick any $x \in B_{s}$ with $x \neq 0$ to get that

$$
A_{\beta} B_{s} \subset B_{s} \Rightarrow \tau_{\beta} \in B_{s} .
$$

Below we shall prove the following:

Lemma 15 If s satisfies

$$
\frac{i s(i)+j \frac{1}{s(j)}}{i+j} \geq e^{a \beta^{\frac{2}{3}}-\beta(2 i+2 j+1)} \text { for all } i \geq 1, j \geq 0,
$$

then $A_{\beta} f_{j} \in B_{s}$ for all $j \geq 1$.

Lemma 15 combined with (4.3-4) shows that

$$
\tau_{\beta} \in \bigcap_{\{s: \text { s satisfies }(4.5)\}} B_{s} .
$$

The r.h.s. of $(4.5)$ is $\leq 1$ when $i+j \geq \frac{a}{2} \beta^{-\frac{1}{3}}$. One therefore easily sees that the following choice of $s$ satisfies (4.5):

$$
\begin{aligned}
s(i) & =1 & & \text { for } i>\frac{a}{2} \beta^{-\frac{1}{3}} \\
& =N \beta^{-\frac{1}{3}} & & \text { for } i \leq \frac{a}{2} \beta^{-\frac{1}{3}}, N \text { large enough. }
\end{aligned}
$$

This proves Lemma 12 .

Proof of Lemma 15. We must show that for all $i, j \geq 1$

$$
\begin{aligned}
0 & \leq s(i)\left(A_{\beta} f_{j}\right)(i) \Leftrightarrow\left(A_{\beta} f_{j}\right)(i+1) \\
& =\sum_{k=1}^{j}\left[s(i) A_{\beta}(i, k) t(k) \Leftrightarrow A_{\beta}(i+1, k \Leftrightarrow 1) t(k \Leftrightarrow 1)\right] \Leftrightarrow A_{\beta}(i+1, j) t(j)
\end{aligned}
$$

(recall from Lemma 4 that $A_{\beta}(i+1,0)=0$ by convention). In order to do so, define

$$
\psi_{i}(j)=\sum_{k=1}^{j}\left[s(i) A_{\beta}(i, k) t(k) \Leftrightarrow A_{\beta}(i+1, k \Leftrightarrow 1) t(k \Leftrightarrow 1)\right] \Leftrightarrow 2 A_{\beta}(i+1, j) t(j) .
$$

The following lemma gives a sufficient criterion for $\psi_{i}(j) \geq 0$, which implies (4.8):

\section{Lemma 16 If}

$$
s(i) A_{\beta}(i, j+1)+\frac{1}{s(j)} A_{\beta}(i+1, j) \Leftrightarrow 2 A_{\beta}(i+1, j+1) \geq 0 \text { for all } i \geq 1, j \geq 0,
$$

then

(i) $j \rightarrow \psi_{i}(j)$ is nondecreasing for all $i \geq 1$

(ii) $\psi_{i}(1) \geq 0$ for all $i \geq 1$. 
Proof. (i) Write out

$$
\begin{aligned}
\psi_{i}(j+1) \Leftrightarrow \psi_{i}(j) & =s(i) A_{\beta}(i, j+1) t(j+1)+A_{\beta}(i+1, j) t(j) \Leftrightarrow 2 A_{\beta}(i+1, j+1) t(j+1) \\
& =t(j+1)\left[s(i) A_{\beta}(i, j+1)+\frac{1}{s(j)} A_{\beta}(i+1, j) \Leftrightarrow 2 A_{\beta}(i+1, j+1)\right] \geq 0 .
\end{aligned}
$$

(ii) Similarly, by (4.10) with $j=0\left(\right.$ since $t(1)=1$ and $\left.A_{\beta}(i+1,0)=0\right)$

$$
\psi_{i}(1)=s(i) A_{\beta}(i, 1) \Leftrightarrow 2 A_{\beta}(i+1,1) \geq 0
$$

To complete the proof of Lemma 15, it remains to rewrite (4.10) in the form of (4.5). Abbreviate $f(i)=\exp \left[a \beta^{\frac{2}{3}} i \Leftrightarrow \beta i^{2}\right]$. Then we have $A_{\beta}(i, j)=f(i+j \Leftrightarrow 1) P(i, j)$. Use Lemma 4 to write

$$
\begin{aligned}
\text { l.h.s. (4.10) }= & f(i+j)\left[s(i) P(i, j+1)+\frac{1}{s(j)} P(i+1, j)\right] \Leftrightarrow 2 f(i+j \Leftrightarrow 1) P(i+1, j+1) \\
= & P(i, j+1)[s(i) f(i+j) \Leftrightarrow f(i+j+1)] \\
& +P(i+1, j)\left[\frac{1}{s(j)} f(i+j) \Leftrightarrow f(i+j+1)\right] .
\end{aligned}
$$

Next use that $P(i, j+1) / P(i+1, j)=i / j$. Then $(4.10)$ is seen to be equivalent to

$$
\frac{i s(i)+j \frac{1}{s(j)}}{i+j} \geq \frac{f(i+j+1)}{f(i+j)} .
$$

\subsection{Proof of Lemma 13}

To prove the upper bound in (3.13), use (2.5)(ii) to get

$$
\begin{aligned}
\lambda(\beta) & =\sum_{i, j} \tau_{\beta}(i) A_{\beta}(i, j) \tau_{\beta}(j) \\
& =\sum_{i, j} \tau_{\beta}(i) e^{e_{\beta}(i, j)} P(i, j) \tau_{\beta}(j) \\
& \leq e^{\frac{1}{4} a^{2} \beta^{\frac{1}{3}}} \sum_{i, j} \tau_{\beta}(i) P(i, j) \tau_{\beta}(j) \\
& \leq e^{\frac{1}{4} a^{2} \beta^{\frac{1}{3}}}
\end{aligned}
$$

where the last inequality follows from $\|P\|_{l^{2}} \leq 1$. This immediately gives the claim.

To prove the lower bound in $(3.13)$, use $(1.3)(\mathrm{i})$ to get that for any $x \in L^{2}\left(\mathbb{R}^{+}\right)$with $\|x\|_{L^{2}}=1$

$$
\beta^{-\frac{1}{3}}[\lambda(\beta) \Leftrightarrow 1] \geq F_{\beta}^{a}(x)
$$

Pick for $x$

$$
x_{\sigma}(u)=\left(\frac{2}{\pi \sigma^{2}}\right)^{\frac{1}{4}} e^{-\frac{u^{2}}{4 \sigma^{2}}}(\sigma>0) .
$$


Now, we know from Lemmas 5-8 that

$$
\lim _{\beta \downarrow 0} F_{\beta}^{a}\left(x_{\sigma}\right)=F^{a}\left(x_{\sigma}\right) .
$$

Hence $\lim \inf _{\beta \downarrow 0} \beta^{-\frac{1}{3}}[\lambda(\beta) \Leftrightarrow 1] \geq F^{a}\left(x_{\sigma}\right)$. Compute

$$
\begin{aligned}
F^{a}\left(x_{\sigma}\right) & =\int_{0}^{\infty}\left\{\left(2 a u \Leftrightarrow 4 u^{2}\right)\left[x_{\sigma}(u)\right]^{2} \Leftrightarrow u\left[x_{\sigma}^{\prime}(u)\right]^{2}\right\} d u \\
& =\left(\frac{2}{\pi \sigma^{2}}\right)^{\frac{1}{2}} \int_{0}^{\infty}\left(2 a u \Leftrightarrow 4 u^{2} \Leftrightarrow \frac{u^{3}}{4 \sigma^{4}}\right) e^{-\frac{u^{2}}{2 \sigma^{2}}} d u \\
& =\left(\frac{8}{\pi}\right)^{\frac{1}{2}} a \sigma \Leftrightarrow 4 \sigma^{2} \Leftrightarrow \frac{1}{(2 \pi)^{\frac{1}{2}} \sigma} .
\end{aligned}
$$

Pick $\sigma=\sigma(a)=\frac{a \vee 1}{(8 \pi)^{\frac{1}{2}}}$ to get the claim.

\section{Analysis of the limit variational problem}

Recall the notation in (1.13)

$$
\begin{aligned}
& X=\left\{x \in L^{2}\left(\mathbb{R}^{+}\right): x \geq 0,\|x\|_{L^{2}}=1\right\} \\
& Y=X \cap C^{1}\left(\mathbb{R}_{0}^{+}\right) \\
& K=K_{C}^{a}=\left\{x \in Y: F^{a}(x) \geq \Leftrightarrow C\right\} .
\end{aligned}
$$

In this section we analyze the limit variational problem appearing in (1.6), i.e.,

$$
\sup _{x \in X} F^{a}(x)
$$

In section 5.1 we show that $x \rightarrow F^{a}(x)$ is upper semicontinuous and $K_{C}^{a}$ is relatively compact in $X$ (in the $L^{2}$-topology). This implies that $F^{a}$ achieves a maximum in $\overline{K_{C}^{a}}=\{x \in X$ : $\left.F^{a}(x) \geq \Leftrightarrow C\right\}\left(\neq \emptyset\right.$ for $C$ large enough). In section 5.2 we show that all maxima of $F^{a}$ in $X$ are solutions of the Sturm-Liouville problem

$$
\mathcal{L}^{a} x=\rho x\left(\rho \in \mathbb{R}, x \in X \cap C^{\infty}\left(\mathbb{R}^{+}\right)\right),
$$

where $\mathcal{L}^{a}$ is defined in $(0.17)$. In section 5.3 we analyze (5.3) and show that it has a unique solution $x^{a}$ satisfying $F^{a}\left(x^{a}\right)>\Leftrightarrow \infty$ and $x^{a}>0$, with corresponding eigenvalue $\rho(a)$. This identifies $x^{a}$ as the unique maximizer of $(5.2)$ and $\rho(a)$ as the maximum. We also study $a \rightarrow x^{a}$ and $a \rightarrow \rho(a)$ to prove the claims that were made in $(0.19)$.

\subsection{Existence of a maximizer of $F^{a}$ in $\overline{K_{C}^{a}}$}

It will be expedient to transform $F^{a}, \mathcal{L}^{a}, K_{C}^{a}$ as follows. Define (recall (1.7))

$$
\begin{aligned}
\hat{F}^{a}(x) & =\Leftrightarrow F^{a}(x)+\left(\frac{a^{2}}{4}+1\right)\|x\|_{L^{2}}^{2} \\
& =\int_{0}^{\infty}\left\{q(u)[x(u)]^{2}+p(u)\left[x^{\prime}(u)\right]^{2}\right\} d u
\end{aligned}
$$


with

$$
\begin{aligned}
p(u) & =u \\
q(u) & =\left(2 u \Leftrightarrow \frac{1}{2} a\right)^{2}+1 .
\end{aligned}
$$

$\hat{F}^{a}$ is the "energy" functional corresponding to the Sturm-Liouville differential operator $\hat{\mathcal{L}}^{a}$ defined by (recall $(0.17)$ )

$$
\begin{aligned}
\left(\hat{\mathcal{L}}^{a} x\right)(u) & =\Leftrightarrow\left(\mathcal{L}^{a} x\right)(u)+\left(\frac{a^{2}}{4}+1\right) x(u) \\
& =q(u) x(u) \Leftrightarrow\left[p(u) x^{\prime}(u)\right]^{\prime} .
\end{aligned}
$$

Define (recall (1.13))

$$
\begin{aligned}
\hat{K}_{C}^{a} & =K_{C-\left(\frac{a^{2}}{4}+1\right)}^{a} \\
& =\left\{x \in Y: \hat{F}^{a}(x) \leq C\right\} .
\end{aligned}
$$

Lemma 17 For every $a \in \mathbb{R}$

(i) $\hat{K}_{C}^{a} \neq \emptyset$ for $C$ large enough

(ii) $\hat{K}_{C}^{a}$ is relatively compact in $L^{2}\left(\mathbb{R}^{+}\right)$for all $C \in \mathbb{R}$

(iii) $x \rightarrow \hat{F}^{a}(x)$ is lower semicontinuous on $X$.

Proof. Standard.

(i) Trivial.

(ii) We check the conditions in Dunford and Schwartz (1964) Theorem IV.8.20.

(a) $\hat{K}_{C}^{a}$ is bounded in $L^{2}\left(\mathbb{R}^{+}\right)$.

(b) By Cauchy-Schwarz

$$
\begin{aligned}
\int_{0}^{\infty}(x(u+v) \Leftrightarrow x(u))^{2} d u & =\int_{0}^{\infty}\left(\int_{u}^{u+v} x^{\prime}(t) d t\right)^{2} \\
& \leq \int_{0}^{\infty} d u[\log (u+v) \Leftrightarrow \log u] \int_{u}^{u+v} d t t\left[x^{\prime}(t)\right]^{2} \\
& =\int_{0}^{\infty} d t t\left[x^{\prime}(t)\right]^{2} I(t, v) 1_{\{t \geq v\}},
\end{aligned}
$$

where

$$
I(t, v)=(t+v) \log \left(1+\frac{v}{t}\right)+(t \Leftrightarrow v) \log \left(1 \Leftrightarrow \frac{v}{t}\right) .
$$

Since $t \rightarrow I(t, v)$ is decreasing and $I(v, v)=2 v \log 2$, it follows that

$$
\lim _{v \downarrow 0} \int_{0}^{\infty}(x(u+v) \Leftrightarrow x(u))^{2} d u=0 \text { uniformly for } x \in \hat{K}_{C}^{a} .
$$

(c) From $p(u) \geq 0$ and $\lim _{u \rightarrow \infty} q(u)=\infty$ follows

$$
\lim _{N \rightarrow \infty} \int_{N}^{\infty} x^{2}(u) d u=0 \text { uniformly for } x \in \hat{K}_{C}^{a} .
$$

Conditions (a)-(c) imply that $\hat{K}_{C}^{a}$ is relatively compact.

(iii) Define

$$
V^{a}=\left\{x \in L^{2}\left(\mathbb{R}^{+}\right): \hat{F}^{a}(x)<\infty\right\} .
$$


On $V^{a}$ define the inner product

$$
\langle x, y\rangle_{V^{a}}=\int_{0}^{\infty}\left\{q(u) x(u) y(u)+p(u) x^{\prime}(u) y^{\prime}(u)\right\} d u .
$$

Then $\left(V^{a},\langle\cdot, \cdot\rangle_{V^{a}}\right)$ is a Hilbert space, $\|x\|_{V^{a}} \geq\|x\|_{L^{2}}$ and

$$
\hat{F}^{a}(x)=\langle x, x\rangle_{V^{a}}=\|x\|_{V^{a}}^{2} .
$$

Thus we must prove that $\lim \inf _{n \rightarrow \infty}\left\|x_{n}\right\|_{V^{a}} \geq\|x\|_{V^{a}}$ for any $x_{n} \rightarrow^{L^{2}} x$.

Let $L=\liminf _{n \rightarrow \infty}\left\|x_{n}\right\|_{V^{a}}$. The case $L=\infty$ being trivial, assume $L<\infty$. Then, by the Banach-Alaoglu theorem (Rudin (1991) Theorem 3.15), there exists a subsequence $\left(x_{n_{k}}\right)$ and a $y \in V^{a}$ such that $L=\lim _{k \rightarrow \infty}\left\|x_{n_{k}}\right\|_{V^{a}}$ and $x_{n_{k}} \rightarrow y$ weakly in $V^{a}(k \rightarrow \infty)$. Hence $L \geq\|y\|_{V^{a}}$ by Fatou. But, by (ii), weak convergence in $V^{a}$ implies strong convergence in $L^{2}\left(\mathbb{R}^{+}\right)$. Hence $x_{n_{k}} \rightarrow^{L^{2}} y$. Together with $x_{n} \rightarrow^{L^{2}} x$ this implies $y=x$ and hence the claim follows.

Incidentally, note from (5.4-5) that $V^{a}$ does not depend on $a$ because it is nothing other than the collection of $x \in L^{2}\left(\mathbb{R}^{+}\right)$for which $\int_{0}^{\infty}\left\{u^{2}[x(u)]^{2}+u\left[x^{\prime}(u)\right]^{2}\right\} d u<\infty$ (recall $(0.18)$ ).

Lemma 17 implies that $\hat{F}^{a}$ achieves a minimum in $\hat{K}_{C}^{a}$ (for $C$ large enough).

\subsection{Characterization of the minimizer(s) of $\hat{F}^{a}$}

Lemma 18 Any minimizer $\bar{x}$ of $\hat{F}^{a}$ in $X$ is a solution of $\hat{\mathcal{L}}^{a} x=\rho x$ for $\rho=\hat{\rho}(a) \in \mathbb{R}$, the minimal eigenvalue of $\hat{\mathcal{L}}^{a}$ in $V^{a}$.

Proof. Standard.

Define $\hat{\rho}(a)$ by

$$
\hat{\rho}(a)=\min _{x \in X} \hat{F}^{a}(x) .
$$

Let $\bar{x} \in V^{a}$ be any minimizer. Then for any $h \in L^{2}\left(\mathbb{R}^{+}\right)$and $\epsilon>0$

$$
\hat{F}^{a}(\bar{x}+\epsilon h) \geq \hat{\rho}(a)\|\bar{x}+\epsilon h\|_{L^{2}}^{2} .
$$

Writing out both sides of (5.16) and using that $\hat{F}^{a}(\bar{x})=\hat{\rho}(a)$, we obtain (see (5.13-14))

$$
2 \epsilon\langle\bar{x}, h\rangle_{V^{a}}+\epsilon^{2}\|h\|_{V^{a}}^{2} \geq \hat{\rho}(a)\left\{2 \epsilon\langle\bar{x}, h\rangle_{L^{2}}+\epsilon^{2}\|h\|_{L^{2}}^{2}\right\} .
$$

Let $\epsilon \downarrow 0$ to obtain

$$
\langle\bar{x}, h\rangle_{V^{a}} \geq \hat{\rho}(a)\langle\bar{x}, h\rangle_{L^{2}} \text { for all } h \in V^{a} .
$$

Replace $h$ by $\Leftrightarrow h$ to get the reverse inequality. Thus

$$
\langle\bar{x}, h\rangle_{V^{a}}=\hat{\rho}(a)\langle\bar{x}, h\rangle_{L^{2}} \text { for all } h \in V^{a} .
$$

Now note that we have from (5.6) and (5.13) after partial integration

$$
\langle\bar{x}, h\rangle_{V^{a}}=\left\langle\bar{x}, \hat{\mathcal{L}}^{a} h\right\rangle_{L^{2}} \text { for all } h \in C_{c}^{2}\left(\mathbb{R}^{+}\right)
$$

It follows from (5.19-20) and the symmetry of $\hat{\mathcal{L}}^{a}$ that $\bar{x}$ is a weak solution of $\hat{\mathcal{L}}^{a} x=\hat{\rho}(a) x$. This in turn implies that $\bar{x}$ is a strong solution.

To see that $\hat{\rho}(a)$ is the minimal eigenvalue of $\hat{\mathcal{L}}^{a}$ in $V^{a}$, note that if $\hat{\mathcal{L}}^{a} x=\rho x$, then by (5.6), (5.13-14) and integration by parts

$$
\hat{F}^{a}(x)=\langle x, x\rangle_{V^{a}}=\left\langle x, \hat{\mathcal{L}}^{a} x\right\rangle_{L^{2}}=\rho\|x\|_{L^{2}}=\rho .
$$




\subsection{Analysis of the Sturm-Liouville problem}

Lemmas 17-18 show that $F^{a}$ has a maximizer in $\overline{K_{C}^{a}}$ and that each maximizer is a solution of $\mathcal{L}^{a} x=\rho x$ for $\rho=\rho(a)$, the maximal eigenvalue of $\mathcal{L}^{a}$ in $V^{a}$ (recall (5.4-7)).

Lemma 19 (i) All solutions of $\mathcal{L}^{a} x=\rho x$ are of the form

$$
x^{a, \rho}(u)=f^{a, \rho}(u)+g^{a, \rho}(u) \log u,
$$

where $f^{a, \rho}$ and $g^{a, \rho}$ are power series with infinite radius of convergence.

(ii) $F^{a}\left(x^{a, \rho}\right)=\Leftrightarrow \infty$ if $g^{a, \rho} \not \equiv 0$.

Proof. (i) Formally substitute $f^{a, \rho}(u)=\sum_{n \geq 0} f_{n} u^{n}$ and $g^{a, \rho}(u)=\sum_{n \geq 0} g_{n} u^{n}$. Then the coefficients are found to satisfy the recurrence relations

$$
\begin{aligned}
& g_{n}=\frac{1}{n^{2}}\left(\rho g_{n-1} \Leftrightarrow 2 a g_{n-2}+4 g_{n-3}\right)(n \geq 1) \\
& f_{n}=\frac{1}{n^{2}}\left(\rho f_{n-1} \Leftrightarrow 2 a f_{n-2}+4 f_{n-3} \Leftrightarrow 2 n g_{n}\right)(n \geq 1)
\end{aligned}
$$

(with $f_{-1}=f_{-2}=g_{-1}=g_{-2}=0$ ). Note that $g^{a, \rho}$ is a solution of (5.3) and that $f^{a, \rho}$ depends on $g^{a, \rho}$. By induction on $n,(5.23)$ is easily shown to give the following bounds:

$$
\begin{aligned}
& \left|f_{n}\right| \leq K_{1}^{n}(n !)^{-\frac{2}{3}}(n \geq 1) \\
& \left|g_{n}\right| \leq K_{2}^{n}(n !)^{-\frac{2}{3}}(n \geq 1)
\end{aligned}
$$

with $K_{1}, K_{2}$ large enough (depending on $\rho, a$ and $f_{0}, g_{0}$ ). This implies that the formal solution exists everywhere.

(ii) Trivial, since $\frac{d}{d u} x^{a, \rho}(u) \sim g_{0} u^{-1}(u \downarrow 0)$ with $g_{0} \neq 0$ implies $F^{a}\left(x^{a, \rho}\right)=\Leftrightarrow \infty$, while $g_{0}=0$ implies that $g_{n} \equiv 0$.

At this stage we know from Lemma 19 that all maximizers of $F^{a}$ are of the form $x^{a, p}(u)=$ $f^{a, \rho}(u)$ and, in particular, are analytic on $\mathbb{R}_{0}^{+}$.

Our next step is to find the asymptotic behavior of the solutions of (5.3) as $u \rightarrow \infty$. This will be needed to get uniqueness of the maximizer.

Lemma $20 \mathcal{L}^{a} x=\rho x$ has two independent solutions $x_{-}^{a, \rho}$ and $x_{+}^{a, \rho}$ satisfying

$$
\lim _{u \rightarrow \infty} u^{-\frac{3}{2}} \log x_{ \pm}^{a, \rho}(u)= \pm \frac{4}{3} .
$$

Proof. We use Coddington and Levinson (1955) Theorem 2.1 page 143-144. Define

$$
\begin{aligned}
& w_{1}(u)=x\left(u^{2}\right) \\
& w_{2}(u)=u^{-2} w_{1}^{\prime}(u) .
\end{aligned}
$$

Then $(5.3)$ can be written as

$$
w^{\prime}(u)=u^{-r} B(u) w(u)
$$

where $r=2$ and

$$
\begin{aligned}
w(u) & =\left(\begin{array}{l}
w_{1}(u) \\
w_{2}(u)
\end{array}\right) \\
B(u) & =\left(\begin{array}{ll}
0 & 1 \\
16 \Leftrightarrow \frac{8 a}{u^{2}}+\frac{4 \rho}{u^{4}} & \Leftrightarrow \frac{3}{u^{3}}
\end{array}\right) .
\end{aligned}
$$


Note that $B(u)=\sum_{n \geq 0} u^{-n} B_{n}\left(B_{0} \neq 0\right)$ is a convergent power series in $u^{-1}$, with $B_{0}$ having eigenvalues $\lambda_{1,2}= \pm 4$. Therefore (5.27) has a formal solution of the form

$$
w(u)=P(u) u^{R} e^{Q(u)},
$$

where $P(u)=\sum_{n=0}^{\infty} u^{-n} P_{n}$ (det $P_{0} \neq 0$ ) is a formal power series in $u^{-1}, R$ is a complex diagonal matrix and $Q=\frac{u^{r+1}}{r+1} Q_{0}+\ldots+u Q_{r}$ is a matrix polynomial with $Q_{i}$ diagonal and $Q_{0}=\operatorname{diag}\left\{\lambda_{1}, \lambda_{2}\right\}$. From the proof of the theorem it follows that $P, Q, R$ can be chosen to be real because $B, \lambda_{1,2}$ are real. On p.151 of Coddington and Levinson (1955) there is the further remark that for every formal solution there exists an actual solution with the same asymptotics.

We see from Lemma 20 that $x_{+}^{a, \rho} \notin L^{2}\left(\mathbb{R}^{+}\right)$and so $(5.3)$ has a unique solution in $L^{2}\left(\mathbb{R}^{+}\right)$ up to multiplicative constants.

Lemma 21 Define

$$
\mathcal{S}_{a}=\left\{\rho \in \mathbb{R}: f^{a, \rho} \in L^{2}\left(\mathbb{R}^{+}\right), f^{a, \rho}(0)=1\right\} .
$$

Then

(i) $\mathcal{S}_{a}$ is countable, bounded from above and has a maximum

(ii) $\rho(a)=\max \mathcal{S}_{a}$ is geometrically simple

(iii) $f^{a, \rho(a)}>0$

(iv) $\forall \rho \in \mathcal{S}_{a}, \rho<\max \mathcal{S}_{a}: f^{a, \rho}$ changes sign in $\mathbb{R}^{+}$.

Proof. Standard Sturm-Liouville theory.

(i),(ii) By Lemma 17(ii), $V^{a}$ is compactly imbedded in $L^{2}\left(\mathbb{R}^{+}\right)$(compare (5.7) and (5.12)). Therefore the eigenfunctions of $\mathcal{L}^{a}$ in $V^{a}$ form an orthogonal basis of $V^{a}$. Since $V^{a}$ is separable, this in turns implies that $\mathcal{S}_{a}$ is countable. We know from Lemmas 19-20 that $\mathcal{L}^{a}$ has a unique eigenvector in $V^{a}$ with eigenvalue $\rho(a)$, i.e., $\rho(a)$ is geometrically simple. Since $\rho(a)=\max _{x \in V^{a}} F^{a}(x)=\max \mathcal{S}^{a}$ by Lemma 18 , we also know that $\mathcal{S}_{a}$ is bounded from above and has a maximum.

(iii) From (1.7) one sees that $F^{a}\left(\left|f^{a, \rho(a)}\right|\right)=F^{a}\left(f^{a, \rho(a)}\right)$. Therefore it follows from the uniqueness of the maximizer that $f^{a, \rho}=\left|f^{a, \rho}\right| \geq 0$. Let $u_{0}=\inf \left\{u>0: f^{a, \rho(a)}(u)=0\right\}>0$. If

$u_{0}<\infty$, then we must have $\frac{d}{d u} f^{a, \rho(a)}\left(u_{0}\right)=0$ and $\frac{d^{2}}{d u^{2}} f^{a, \rho(a)}\left(u_{0}\right)>0$. However, this contra$\operatorname{dicts}\left(\mathcal{L}^{a} f^{a, \rho(a)}\right)(u)=\rho(a) f^{a, \rho(a)}(u)$ at the point $u=u_{0}$ (see $(0.17)$ ).

(iv) This follows from (iii) and the fact that the eigenfunctions of $\mathcal{L}^{a}$ in $V^{a}$ form an orthogonal basis.

Lemmas 17-18 and 21 show that Assumptions 3(i), (ii) in proposition 2 hold.

\subsection{Dependence on a}

The maximal eigenvalue and eigenvector of $(0.17-18)$ are

$$
\begin{aligned}
\rho(a) & =\max \mathcal{S}_{a} \\
x^{a} & =\frac{f^{a, \rho(a)}}{\left\|f^{a, \rho(a)}\right\|_{L^{2}}} .
\end{aligned}
$$

We can now prove the following properties: 
Lemma 22 (i) $a \rightarrow \rho(a)$ and $a \rightarrow x^{a}$ are analytic

(ii) $a \rightarrow \rho(a)$ is strictly increasing and strictly convex on $\mathbb{R}$

(iii) $\rho(0)<0, \lim _{a \uparrow \infty} \rho(a)=\infty$ and $\lim _{a \downarrow-\infty} \rho(a)=\Leftrightarrow \infty$.

Proof. (i) We give the proof by applying Crandall and Rabinowitz (1973) Lemma 1.3 in the following setting. Pick $a \in \mathbb{R}$ and consider the Hilbert space $\left(V,\langle\cdot, \cdot\rangle_{V}\right)$ with $V=V^{0}$. Then, from (5.5-6) and (5.13),

$$
\begin{aligned}
& \left\langle x^{a}, y\right\rangle_{V^{a}}=\left\langle\mathcal{L}^{a} x^{a}, y\right\rangle_{L^{2}}=\rho(a)\left\langle x^{a}, y\right\rangle_{L^{2}} \\
& \left\langle x^{a}, y\right\rangle_{V^{a}}=\left\langle x^{a}, y\right\rangle_{V} \Leftrightarrow 2 a b\left(x^{a}, y\right)+\frac{a^{2}}{4}\left\langle x^{a}, y\right\rangle_{L^{2}},
\end{aligned}
$$

where $b: V \times V \rightarrow \mathbb{R}$ is the bilinear form defined by

$$
b(x, y)=\int_{0}^{\infty} u x(u) y(u) d u .
$$

For every $x \in V$ the functional $y \rightarrow b(x, y)$ is continuous and linear. Hence it follows from the Riesz representation theorem (Rudin (1987) Theorem 6.19) that there exists a unique linear operator $B: V \rightarrow V$ such that

$$
b(x, y)=\langle B x, y\rangle_{V} \text { for all } x, y \in V .
$$

$B$ is symmetric because $b$ is. $B$ is bounded because

$$
\begin{aligned}
\|B x\|_{V}^{2} & =b(x, B x) \\
& \leq\left(\int_{0}^{\infty} u^{2} x^{2}(u) d u\right)^{\frac{1}{2}}\|B x\|_{L^{2}} \\
& \leq \frac{1}{2}\|x\|_{V}\|B x\|_{L^{2}} \\
& \leq \frac{1}{2}\|x\|_{V}\|B x\|_{V}
\end{aligned}
$$

(see (5.5) and (5.13)), so that $\|B x\|_{V} \leq \frac{1}{2}\|x\|_{V}$. To see that $B$ is compact, let $\left(x_{n}\right)$ be a bounded sequence in $V$. Then, by Lemma 17 (ii), there exists a subsequence $\left(x_{n_{k}}\right)$ and an $x \in V$ such that $x_{n_{k}} \rightarrow{ }^{L^{2}} x(k \rightarrow \infty)$. Hence, as in (5.35),

$$
\begin{aligned}
\left\|B x_{n_{k}} \Leftrightarrow B x\right\|_{V}^{2} & =b\left(x_{n_{k}} \Leftrightarrow x, B\left(x_{n_{k}} \Leftrightarrow x\right)\right) \\
& \leq\left\|x_{n_{k}} \Leftrightarrow x\right\|_{L^{2}} \frac{1}{2}\left\|B\left(x_{n_{k}} \Leftrightarrow x\right)\right\|_{V} \\
& \leq\left\|x_{n_{k}} \Leftrightarrow x\right\|_{L^{2}} \frac{1}{4}\left\|x_{n_{k}} \Leftrightarrow x\right\|_{V} \\
& \rightarrow 0(k \rightarrow \infty) .
\end{aligned}
$$

In the same manner we can prove that there exists a unique linear, symmetric and compact operator $C: V \rightarrow V$ such that

$$
\langle x, y\rangle_{L^{2}}=\langle C x, y\rangle_{V} \text { for all } x, y \in V .
$$


Now rewrite (5.32) as follows

$$
\left\langle\left[I d \Leftrightarrow 2 a B \Leftrightarrow\left(\rho(a) \Leftrightarrow \frac{a^{2}}{4}\right) C\right] x^{a}, y\right\rangle_{V}=0 \text { for all } y \in V .
$$

Hence, $\left(V,\langle\cdot, \cdot\rangle_{V}\right)$ being a Hilbert space, we have

$$
\begin{aligned}
& x^{a} \text { is a } C \text {-eigenfunction of } I d \Leftrightarrow 2 a B \\
& \text { with (largest) eigenvalue } \rho(a) \Leftrightarrow \frac{a^{2}}{4} .
\end{aligned}
$$

Next note that $a \rightarrow I d \Leftrightarrow 2 a B$ is analytic in the operator norm. Therefore, to get the claim from Crandall and Rabinowitz (1973) Lemma 1.3, it suffices to check that $\rho(a) \Leftrightarrow \frac{a^{2}}{4}$ is a $C$-simple eigenvalue of $I d \Leftrightarrow 2 a B$, i.e.,

(a) $\operatorname{dim}\left(N\left(A^{a}\right)\right)=\operatorname{codim}\left(R\left(A^{a}\right)\right)=1$

(b) $C x^{a} \notin R\left(A^{a}\right)$,

where $A^{a}=I d \Leftrightarrow 2 a B \Leftrightarrow\left(\rho(a) \Leftrightarrow \frac{a^{2}}{4}\right) C$ and $N\left(A^{a}\right), R\left(A^{a}\right)$ denote the null space resp. range of $A^{a}$.

We have $\operatorname{dim}\left(N\left(A^{a}\right)\right)=1$ because of Lemma 21 (ii). Moreover, because $2 a B+\left(\rho(a) \Leftrightarrow \frac{a^{2}}{4}\right) C$ is compact we have $\operatorname{dim}\left(N\left(A^{a}\right)\right)=\operatorname{codim}\left(R\left(A^{a}\right)\right)$ (Rudin (1991) Theorem 4.25). This proves (a). To prove (b), first use that $A^{a}$ is symmetric and bounded to get that $N\left(A^{a}\right)=R\left(A^{a}\right)^{\perp}$ (the orthogonal complement of $R\left(A^{a}\right)$ ) and $R\left(A^{a}\right)=\overline{R\left(A^{a}\right)}$ (Rudin (1991) Theorems 4.12 and 4.23). Since $\overline{R\left(A^{a}\right)}=R\left(A^{a}\right)^{\perp \perp}$, it follows that $N\left(A^{a}\right)^{\perp}=R\left(A^{a}\right)$. Hence (b) is equivalent to $\left\langle C x^{a}, x^{a}\right\rangle_{V} \neq 0$. But $\left\langle C x^{a}, x^{a}\right\rangle_{V}=\left\langle x^{a}, x^{a}\right\rangle_{L^{2}}=1$ by $(5.37)$.

(ii) Because

$$
\rho(a)=\sup _{x \in X} F^{a}(x)
$$

with unique maximizer $x=f^{a, \rho(a)}$, we immediately see from (1.7) that

$$
\frac{\rho(a+\epsilon) \Leftrightarrow \rho(a)}{\epsilon} \geq \int_{0}^{\infty} 2 u\left[f^{a, \rho(a)}(u)\right]^{2} d u>0
$$

(pick $\left\|f^{a, \rho(a)}\right\|_{L^{2}}=1$ ). This demonstrates that $\rho^{\prime}(a)$ is everywhere strictly positive. Moreover, since $a \rightarrow F^{a}(x)$ is linear for every $x$ we have from (5.40) that $a \rightarrow \rho(a)$ is convex. Because of analyticity, it follows that either $a \rightarrow \rho(a)$ is strictly convex or $\rho(a)=C_{1} a+C_{2}$. However, the latter is impossible because of Lemma 13.

(iii) Trivial. Let $\epsilon \rightarrow \pm \infty$ in (5.41) or else see (1.7).

\section{Proof of Theorems 4-7}

We can now collect the results from sections 2-5 and give the proofs of our theorems in section 0.4 .

Proof of Theorem 5. Combine Propositions 1-3 with (1.13). The proof of Proposition 3 was given in Lemma 1 and in sections 2, 3 and 5 .

\section{Proof of Theorems 4 and 6.}

1. $r^{*}(\beta) \sim a^{*} \beta^{\frac{2}{3}}$. 
According to $(0.13), r^{*}(\beta)$ is defined as the unique solution of

$$
\lambda(r, \beta)=1 .
$$

From (0.20) we know that for every $a \in \mathbb{R}$

$$
\beta^{-\frac{1}{3}}\left[\lambda\left(a \beta^{\frac{2}{3}}, \beta\right) \Leftrightarrow 1\right] \rightarrow \rho(a) .
$$

Let $a^{*}>0$ be the solution of $\rho(a)=0$ (see Lemma 22). Now, because $r \rightarrow \lambda(r, \beta)$ is increasing (as is obvious from $(0.10)$ ), we have for every $\epsilon>0$

$$
\begin{aligned}
& \lambda(r, \beta) \geq 1+\beta^{\frac{1}{3}} \rho\left(a^{*}+\epsilon\right)+o\left(\beta^{\frac{1}{3}}\right) \text { for } r \geq\left(a^{*}+\epsilon\right) \beta^{\frac{2}{3}} \\
& \lambda(r, \beta) \leq 1+\beta^{\frac{1}{3}} \rho\left(a^{*} \Leftrightarrow \epsilon\right)+o\left(\beta^{\frac{1}{3}}\right) \text { for } r \leq\left(a^{*} \Leftrightarrow \epsilon\right) \beta^{\frac{2}{3}} .
\end{aligned}
$$

Since $\rho\left(a^{*} \Leftrightarrow \epsilon\right)<0<\rho\left(a^{*}+\epsilon\right)$ for every $\epsilon>0$ (see Lemma 22(ii)), (6.1) combined with (6.3) implies

$$
\left(a^{*} \Leftrightarrow \epsilon\right) \beta^{\frac{2}{3}} \leq r^{*}(\beta) \leq\left(a^{*}+\epsilon\right) \beta^{\frac{2}{3}} \text { for } \beta \text { small enough. }
$$

Let $\epsilon \downarrow 0$ to get the claim.

2. $\theta^{*}(\beta) \sim b^{*} \beta^{\frac{1}{3}}$.

According to $(0.14), \theta^{*}(\beta)$ is defined as

$$
\frac{1}{\theta^{*}(\beta)}=\left[\frac{\partial}{\partial r} \lambda(r, \beta)\right]_{r=r^{*}(\beta)}
$$

Define

$$
\xi(r, \beta)=\frac{\frac{\partial}{\partial r} \lambda(r, \beta)}{\lambda(r, \beta)}=\frac{\partial}{\partial r} \log \lambda(r, \beta) .
$$

Because $r \rightarrow \lambda(r, \beta)$ is increasing and log-convex (see footnote 6 ), we have that for all $h, \beta>0$ and $a \in \mathbb{R}$

$$
\begin{aligned}
& \xi\left(a \beta^{\frac{2}{3}}, \beta\right) \leq \frac{1}{h \beta^{\frac{2}{3}}}\left[\log \lambda\left((a+h) \beta^{\frac{2}{3}}, \beta\right) \Leftrightarrow \log \lambda\left(a \beta^{\frac{2}{3}}, \beta\right)\right] \\
& \xi\left(a \beta^{\frac{2}{3}}, \beta\right) \geq \frac{1}{h \beta^{\frac{2}{3}}}\left[\log \lambda\left(a \beta^{\frac{2}{3}}, \beta\right) \Leftrightarrow \log \lambda\left((a \Leftrightarrow h) \beta^{\frac{2}{3}}, \beta\right)\right] .
\end{aligned}
$$

Together with (6.2) this gives

$$
\begin{aligned}
& \limsup _{\beta \downarrow 0} \beta^{\frac{1}{3}} \xi\left(a \beta^{\frac{2}{3}}, \beta\right) \leq \frac{\rho(a+h) \Leftrightarrow \rho(a)}{h} \\
& \liminf _{\beta \downarrow 0} \beta^{\frac{1}{3}} \xi\left(a \beta^{\frac{2}{3}}, \beta\right) \geq \frac{\rho(a) \Leftrightarrow \rho(a \Leftrightarrow h)}{h} .
\end{aligned}
$$

Let $h \downarrow 0$ to get (use Lemma 22)

$$
\lim _{\beta \downarrow 0} \beta^{\frac{1}{3}} \xi\left(a \beta^{\frac{2}{3}}, \beta\right)=\rho^{\prime}(a) .
$$

Next, because $r \rightarrow \xi(r, \beta)$ is increasing we have, via (6.4), for $\beta$ small enough

$$
\begin{aligned}
& \xi\left(r^{*}(\beta), \beta\right) \leq \xi\left(\left(a^{*}+\epsilon\right) \beta^{\frac{2}{3}}, \beta\right)=\beta^{-\frac{1}{3}} \rho^{\prime}\left(a^{*}+\epsilon\right)+o\left(\beta^{-\frac{1}{3}}\right) \\
& \xi\left(r^{*}(\beta), \beta\right) \geq \xi\left(\left(a^{*} \Leftrightarrow \epsilon\right) \beta^{\frac{2}{3}}, \beta\right)=\beta^{-\frac{1}{3}} \rho^{\prime}\left(a^{*} \Leftrightarrow \epsilon\right)+o\left(\beta^{-\frac{1}{3}}\right) .
\end{aligned}
$$


Since (recall that $\lambda\left(r^{*}(\beta), \beta\right)=1$ )

$$
\frac{1}{\theta^{*}(\beta)}=\xi\left(r^{*}(\beta), \beta\right),
$$

it follows that

$$
\rho^{\prime}\left(a^{*} \Leftrightarrow \epsilon\right) \leq \frac{1}{\beta^{-\frac{1}{3}} \theta^{*}(\beta)} \leq \rho^{\prime}\left(a^{*}+\epsilon\right) \text { for } \beta \text { small enough. }
$$

Let $\epsilon \downarrow 0$ to get the claim with $\frac{1}{b^{*}}=\rho^{\prime}(a)$.

3. $\beta^{-\frac{1}{6}} \tau_{r^{*}(\beta), \beta}\left(\left\lceil\cdot \beta^{-\frac{1}{3}}\right\rceil\right) \rightarrow{ }^{L^{2}} x^{a^{*}}(\cdot)$.

Put $a^{*}(\beta)=\beta^{-\frac{2}{3}} r^{*}(\beta)$. Then, similarly as in Lemma 1 ,

$$
\beta^{-\frac{1}{6}} \tau_{r *(\beta), \beta}\left(\left\lceil\cdot \beta^{-\frac{1}{3}}\right\rceil\right) \text { is the unique maximizer of } F_{\beta}^{a^{*}(\beta)} \text {, }
$$

where the parameter $a$ is replaced by $a^{*}(\beta)$.

Lemma 23 Assumptions (1)-(3) in Proposition 2 hold for the following choice replacing (1.13):

$$
\begin{aligned}
K & =K_{C}^{a^{*}}(C \text { sufficiently large }) \\
G_{\beta} & =F_{\beta}^{a^{*}(\beta)} \\
G & =F^{a^{*}}
\end{aligned}
$$

Proof. The point is that $\lim _{\beta \downarrow 0} a^{*}(\beta)=a^{*}$. It is trivial to check that all estimates in sections 2 and 3 remain valid when the fixed parameter $a$ is replaced by $a+o(1)(\beta \downarrow 0)$. See, in particular, the proofs of Lemmas 5, 6, 11-13.

The claim in $\mathbf{3}$ now follows from Proposition 2.

4. $\beta^{-\frac{1}{3}} \mu_{\beta}^{*}\left(\left\lceil\cdot \beta^{-\frac{1}{3}}\right\rceil\right) \rightarrow^{L^{1}} \frac{1}{2}\left[x^{a^{*}}\left(\frac{1}{2} \cdot\right)\right]^{2}$.

The proof is in Steps 1-2 below.

Abbreviate $A_{\beta}=A_{r^{*}(\beta), \beta}$ and $\tau_{\beta}=\tau_{r^{*}(\beta), \beta}$. According to $(0.14)$

$$
\mu_{\beta}^{*}(k)=\sum_{\substack{i, j \\ i+j-1=k}} \tau_{\beta}(i) A_{\beta}(i, j) \tau_{\beta}(j) .
$$

STEP 1 There exists $c$ such that

$$
\int_{N}^{\infty}\left|\beta^{-\frac{1}{3}} \mu_{\beta}^{*}\left(\left\lceil u \beta^{-\frac{1}{3}}\right\rceil\right) \Leftrightarrow \frac{1}{2}\left[x^{a^{*}}\left(\frac{1}{2} u\right)\right]^{2}\right| d u \leq c N^{-2} \text { for } \beta \text { small enough. }
$$


Proof. Estimate (recall that $\lambda\left(r^{*}(\beta), \beta\right)=1$ )

$$
\begin{aligned}
\int_{N}^{\infty} \beta^{-\frac{1}{3}} \mu_{\beta}^{*}\left(\left\lceil u \beta^{-\frac{1}{3}}\right\rceil\right) d u & =\sum_{k \geq N \beta^{-\frac{1}{3}}} \mu_{\beta}^{*}(k) \\
& =\sum_{i, j: i+j-1 \geq N \beta^{-\frac{1}{3}}} \tau_{\beta}(i) A_{\beta}(i, j) \tau_{\beta}(j) \\
& \leq 2 \sum_{i \geq \frac{1}{2} N \beta^{-\frac{1}{3}}} \tau_{\beta}^{2}(i) \\
& \leq 8 N^{-2} \beta^{\frac{2}{3}} \sum_{i \geq 1} i^{2} \tau_{\beta}^{2}(i) \\
& \leq 8 C_{1} N^{-2}
\end{aligned}
$$

The last inequality is Lemma 11(i). Furthermore,

$$
\int_{N}^{\infty} \frac{1}{2}\left[x^{a^{*}}\left(\frac{1}{2} u\right)\right]^{2} d u \leq 4 N^{-2} \int_{N}^{\infty} u^{2}\left[x^{a^{*}}\left(\frac{1}{2} u\right)\right]^{2} d u .
$$

Since $x^{a^{*}} \in K_{C}^{a^{*}}$, the integral in the r.h.s. is finite and so the claim follows.

STEP $2 \lim _{\beta \downarrow 0} \int_{0}^{N}\left|\beta^{-\frac{1}{3}} \mu_{\beta}^{*}\left(\left\lceil u \beta^{-\frac{1}{3}}\right\rceil\right) \Leftrightarrow \frac{1}{2}\left[x^{a^{*}}\left(\frac{1}{2} u\right)\right]^{2}\right| d u=0$ for every fixed $N$.

Proof. Use the triangle inequality to split the integal into three parts:

$$
\int_{0}^{N}\left|\beta^{-\frac{1}{3}} \mu_{\beta}^{*}\left(\left\lceil u \beta^{-\frac{1}{3}}\right\rceil\right) \Leftrightarrow \frac{1}{2}\left[x^{a^{*}}\left(\frac{1}{2} u\right)\right]^{2}\right| d u \leq I_{\beta}^{1, N}+I_{\beta}^{2, N}+I_{\beta}^{3, N}
$$

with

$$
\begin{aligned}
I_{\beta}^{1, N} & =\beta^{\frac{1}{3}} \sum_{i, j: i+j-1 \leq N \beta^{-\frac{1}{3}}}\left|\bar{\tau}_{\beta}\left(i \beta^{\frac{1}{3}}\right) \Leftrightarrow x^{a^{*}}\left(i \beta^{\frac{1}{3}}\right)\right| A_{\beta}(i, j) x^{a^{*}}\left(j \beta^{\frac{1}{3}}\right) \\
I_{\beta}^{2, N} & =\beta^{\frac{1}{3}} \sum_{i, j: i+j-1 \leq N \beta^{-\frac{1}{3}}} \bar{\tau}_{\beta}\left(i \beta^{\frac{1}{3}}\right) A_{\beta}(i, j)\left|\bar{\tau}_{\beta}\left(j \beta^{\frac{1}{3}}\right) \Leftrightarrow x^{a^{*}}\left(j \beta^{\frac{1}{3}}\right)\right| \\
I_{\beta}^{3, N} & =\int_{0}^{N}\left|\sum_{i, j: i+j-1=\left\lceil u \beta^{-\frac{1}{3}}\right\rceil} x^{a^{*}}\left(i \beta^{\frac{1}{3}}\right) A_{\beta}(i, j) x^{a^{*}}\left(j \beta^{\frac{1}{3}}\right) \Leftrightarrow \frac{1}{2}\left[x^{a^{*}}\left(\frac{1}{2} u\right)\right]^{2}\right| d u .
\end{aligned}
$$

Here $\bar{\tau}_{\beta}$ is the scaled form of $\tau_{\beta}$ given by the same relation as (3.1).

For $I_{\beta}^{1, N}$ use Cauchy-Schwarz and (2.5)(ii) to estimate

$$
\begin{aligned}
I_{\beta}^{1, N} \leq & \beta^{\frac{1}{3}}\left(\sum_{i, j: i+j-1 \leq N \beta^{-\frac{1}{3}}}\left[\bar{\tau}_{\beta}\left(i \beta^{\frac{1}{3}}\right) \Leftrightarrow x^{a^{*}}\left(i \beta^{\frac{1}{3}}\right)\right]^{2} A_{\beta}(i, j)\right)^{\frac{1}{2}} \\
& \times\left(\sum_{i, j: i+j-1 \leq N \beta^{-\frac{1}{3}}}\left[x^{a^{*}}\left(j \beta^{\frac{1}{3}}\right)\right]^{2} A_{\beta}(i, j)\right)^{\frac{1}{2}} \\
\leq & e^{\frac{1}{4} a^{2} \beta^{\frac{1}{3}}}\left(\beta^{\frac{1}{3}} \sum_{i \leq N \beta^{-\frac{1}{3}}}\left[\bar{\tau}_{\beta}\left(i \beta^{\frac{1}{3}}\right) \Leftrightarrow x^{a^{*}}\left(i \beta^{\frac{1}{3}}\right)\right]^{2}\right)^{\frac{1}{2}} \\
& \times\left(\beta^{\frac{1}{3}} \sum_{\left.j \leq N \beta^{-\frac{1}{3}}\left[x^{a^{*}}\left(j \beta^{\frac{1}{3}}\right)\right]^{2}\right)^{\frac{1}{2}} .}\right.
\end{aligned}
$$

Define $\bar{x}_{\beta}^{a^{*}}$ by $\bar{x}_{\beta}^{a^{*}}(u)=x^{a^{*}}\left(i \beta^{\frac{1}{3}}\right)$ for $(i \Leftrightarrow 1) \beta^{\frac{1}{3}} \leq u \leq i \beta^{\frac{1}{3}}(i \geq 1)$, in analogy with (3.1). Then (6.21) becomes

$$
I_{\beta}^{1, N} \leq e^{\frac{1}{4} a^{2} \beta^{\frac{1}{3}}}\left\|\bar{\tau}_{\beta} \Leftrightarrow \bar{x}_{\beta}^{a^{*}}\right\|_{L^{2}[0, N]}\left\|\bar{x}_{\beta}^{a^{*}}\right\|_{L^{2}[0, N]}
$$


Now let $\beta \downarrow 0$ and use that $\bar{\tau}_{\beta} \rightarrow{ }^{L^{2}} x^{a^{*}}$ and $\bar{x}_{\beta}^{a^{*}} \rightarrow^{L^{2}} x^{a^{*}}$ to get $\lim \sup _{\beta \downarrow 0} I_{\beta}^{1, N}=0$. The same argument gives that $\lim \sup _{\beta \downarrow 0} I_{\beta}^{2, N}=0$.

To estimate $I_{\beta}^{3, N}$, we use the mean value theorem to expand $x^{a^{*}}\left(i \beta^{\frac{1}{3}}\right)$ and $x^{a^{*}}\left(j \beta^{\frac{1}{3}}\right)$ around $\frac{1}{2} u$. Namely

$$
\begin{aligned}
I_{\beta}^{3, N}= & \int_{0}^{N} \mid \sum_{i, j: i+j-1=\left\lceil u \beta^{-\frac{1}{3}}\right\rceil}\left\{x^{a^{*}}\left(\frac{1}{2} u\right)+\left(i \beta^{\frac{1}{3}} \Leftrightarrow \frac{1}{2} u\right) \frac{d}{d u} x^{a^{*}}(\xi)\right\} \\
& \times A_{\beta}(i, j)\left\{x^{a^{*}}\left(\frac{1}{2} u\right)+\left(j \beta^{\frac{1}{3}} \Leftrightarrow \frac{1}{2} u\right) \frac{d}{d u} x^{a^{*}}(\eta)\right\} \Leftrightarrow \frac{1}{2}\left[x^{a^{*}}\left(\frac{1}{2} u\right)\right]^{2} \mid d u
\end{aligned}
$$

with $\xi, \eta$ between $\frac{1}{2} u$ and $i \beta^{\frac{1}{3}}$ resp. $j \beta^{\frac{1}{3}}$. Next note that $x^{a^{*}}(u),\left|\frac{d}{d u} x^{a^{*}}(u)\right| \leq M<\infty$ for all $u \in \mathbb{R}^{+}$. Hence

$$
\begin{aligned}
I_{\beta}^{3, N} \leq & M^{2} \int_{0}^{N}\left|\sum_{i, j: i+j-1=\left\lceil u \beta^{-\frac{1}{3}}\right\rceil} A_{\beta}(i, j) \Leftrightarrow \frac{1}{2}\right| d u \\
& +2 M^{2} \int_{0}^{N} \sum_{i, j: i+j-1=\left\lceil u \beta^{-\frac{1}{3}}\right\rceil}\left\{\left|i \beta^{\frac{1}{3}} \Leftrightarrow \frac{1}{2} u\right|+\left|i \beta^{\frac{1}{3}} \Leftrightarrow \frac{1}{2} u\right|^{2}\right\} A_{\beta}(i, j) .
\end{aligned}
$$

Next we insert $A_{\beta}=e^{e_{\beta}} P$ and use that (recall (2.4) and $(0.11)$ )

$$
\begin{gathered}
\left|e_{\beta}(i, j)\right| \leq(|a|+N) N \beta^{\frac{1}{3}} \text { for } i, j \leq N \beta^{-\frac{1}{3}} \\
\sum_{\substack{i, j \\
i+j-1=k}} P(i, j)=\frac{1}{2}(k \geq 1) \\
\sum_{\substack{i, j \\
i+j-1=k}}\left(i \Leftrightarrow \frac{1}{2} k\right)^{2} P(i, j)=\frac{1}{2}\left(\frac{1}{4} k+1\right)(k \geq 1)
\end{gathered}
$$

Then (6.24) yields

$$
I_{\beta}^{3, N} \leq M^{2} N \frac{1}{2}\left(e^{(|a|+N) N \beta^{\frac{1}{3}}} \Leftrightarrow 1\right)+2 M^{2} e^{\frac{1}{4} a^{2} \beta^{\frac{1}{3}}} \int_{0}^{N} d u\left\{\left(z_{\beta}(u)\right)^{\frac{1}{2}}+z_{\beta}(u)\right\}
$$

where

$$
z_{\beta}(u)=\beta^{\frac{2}{3}} \sum_{i, j: i+j-1=\left\lceil u \beta^{-\frac{1}{3}}\right\rceil}\left(i \Leftrightarrow \frac{1}{2} u \beta^{-\frac{1}{3}}\right)^{2} P(i, j)=\mathcal{O}\left(\beta^{\frac{1}{3}}\right) .
$$

Let $\beta \downarrow 0$ to get $\lim \sup _{\beta \downarrow 0} I_{\beta}^{3, N}=0$.

Steps 1-2 prove the claim in 4.

Results 1-4 complete the proof of Theorems 4 and 6 .

Proof of Theorem 7. The asymptotic behavior of $x^{a^{*}}$ in (iii) was proved in Lemma 20 (pick $a=a^{*}$ and $\rho=0$ ). To prove (i) and (ii), we recall that $x^{a^{*}}$ solves (see $(0.17)$ )

$$
0=\left(\mathcal{L}^{a^{*}} x\right)(u)=\left(2 a^{*} u \Leftrightarrow 4 u^{2}\right) x(u)+\left[u x^{\prime}\right]^{\prime}(u)
$$

and has a power series representation (see (5.23))

$$
\begin{aligned}
& x^{a^{*}}(u)=\sum_{n \geq 0} x_{n} u^{n} \\
& x_{n}=\frac{1}{n^{2}}\left(\Leftrightarrow 2 a^{*} x_{n-2}+4 x_{n-3}\right)(n \geq 1) \\
& x_{-1}=x_{-2}=0 .
\end{aligned}
$$


We observe that $u \rightarrow 2 a^{*} u \Leftrightarrow 4 u^{2}$ changes $\operatorname{sign}$ from positive to negative at $u=\frac{1}{2} a^{*}$. Since $x^{a^{*}}(u)>0$ for all $u \geq 0$, it follows from $(6.30)$ that $u \rightarrow u \frac{d}{d u} x^{a^{*}}(u)$ is unimodal with a minimum at $u=\frac{1}{2} a^{*}$. It is clear that $u \frac{d}{d u} x^{a^{*}}(u) \rightarrow 0$ as $u \downarrow 0$. By the unimodality we must have that $u \frac{d}{d u} x^{a^{*}}(u) \rightarrow c$ as $u \rightarrow \infty$. However, $c$ must be 0 otherwise $\int_{0}^{\infty} u\left[\frac{d}{d u} x^{a^{*}}(u)\right]^{2} d u=\infty$, which is impossible since $F^{a^{*}}\left(x^{a^{*}}\right)=\rho\left(a^{*}\right)=0>\Leftrightarrow \infty$ (see (1.7)). Thus we conclude that $u \frac{d}{d u} x^{a^{*}}(u)<0$ for all $u>0$, which implies that $u \rightarrow x^{a^{*}}(u)$ is strictly decreasing.

To prove (iv), use $(0.15)$ to write

$$
\begin{aligned}
\frac{\beta^{\frac{1}{3}}}{\theta^{*}(\beta)} & =\beta^{\frac{1}{3}} \sum_{i}(2 i \Leftrightarrow 1) \tau_{r^{*}(\beta), \beta}^{2}(i) \\
& =\int_{0}^{\infty} 2 u\left\{\beta^{-\frac{1}{6}} \tau_{r^{*}(\beta), \beta}\left(\left\lceil u \beta^{-\frac{1}{3}}\right\rceil\right)\right\}^{2} d u .
\end{aligned}
$$

As $\beta \downarrow 0$ the 1.h.s. tends to $\frac{1}{b^{*}}$. Thus we must show that the r.h.s. tends to $\int_{0}^{\infty} 2 u\left[x^{a^{*}}(u)\right]^{2} d u$. To prove this claim, first note that

$$
\begin{aligned}
\int_{N}^{\infty} 2 u\left\{\beta^{-\frac{1}{6}} \tau_{r^{*}(\beta), \beta}\left(\left\lceil u \beta^{-\frac{1}{3}}\right\rceil\right)\right\}^{2} d u & =\beta^{\frac{1}{3}} \sum_{i \geq N \beta^{-\frac{1}{3}}}(2 i \Leftrightarrow 1) \tau_{r^{*}(\beta), \beta}^{2}(i) \\
& \leq \frac{2}{N} \beta^{\frac{2}{3}} \sum_{i \geq N \beta^{-\frac{1}{3}}} i^{2} \tau_{r^{*}(\beta), \beta}^{2}(i) \\
& \leq \frac{2}{N} C_{1} \text { for } \beta \text { sufficiently small }
\end{aligned}
$$

where we use Lemma 11(i). Similarly $\int_{N}^{\infty} 2 u\left[x^{a^{*}}(u)\right]^{2} d u \leq \frac{2}{N} \int_{N}^{\infty} u^{2}\left[x^{a^{*}}(u)\right]^{2} d u=o\left(N^{-1}\right)$ as $N \rightarrow \infty$. Next, recall 3 in the proof of Theorems 4 and 6 to see that

$$
\lim _{\beta \downarrow 0} \int_{0}^{N} 2 u\left\{\beta^{-\frac{1}{6}} \tau_{r^{*}(\beta), \beta}\left(\left\lceil u \beta^{-\frac{1}{3}}\right\rceil\right)\right\}^{2} d u=\int_{0}^{N} 2 u\left[x^{a^{*}}(u)\right]^{2} d u \text { for all } N \text {. }
$$

Let $N \rightarrow \infty$ to get the claim.

\section{Acknowledgments}

We thank Ph. Clément for help with the functional analysis in this paper and J.-B. Baillon, E. Balder, J. Duistermaat and R. Gill for useful remarks during various stages of this work. We thank D. Brydges, G Slade and J. Westwater for their contribution to section 0.6 clarifying the connection between the Domb-Joyce model and the Edwards model.

\section{References}

[1] H. Attouch, Variational Convergence for Functions and Operators, Pitman, Boston 1984.

[2] D.C. Brydges and T. Spencer, Self-avoiding walk in 5 or more dimensions, Commun. Math. Phys. 97, 125-148, 1985.

[3] D.C. Brydges and G. Slade, The diffusive phase of a model of self interacting walks. Preprint 1994. 
[4] E.A. Coddington and N. Levinson, Theory of Ordinary Differential Equations, McGrawHill, New York 1955.

[5] M.G. Crandall and P.H. Rabinowitz, Bifurcations, perturbations of simple eigenvalues, and linearized stability, Arch. Rat. Mech. Anal. 52, 162-180, 1973.

[6] N. Dunford and J.T. Schwartz, Linear Operators. Part I: General Theory, $2^{\text {nd }}$ printing, Interscience Publishers, New York 1964.

[7] A. Greven and F. den Hollander, A variational characterization of the speed of a onedimensional self-repellent random walk, Ann. Appl. Probab. 3, 1067-1099, 1993.

[8] T. Hara and G. Slade, Self-avoiding walk in five or more dimensions. I. The critical behaviour, Commun. Math. Phys. 147, 101-136, 1992.

[9] T. Hara and G. Slade, The lace expansion for self-avoiding walk in five or more dimensions, Reviews in Math. Phys. 4, 235-327, 1992.

[10] N. Madras and G. Slade, The Self-Avoiding Walk, Birkhäuser, Boston 1993.

[11] P. Révész, Random Walks in Random and Non-Random Environments, World Scientific, Singapore 1990.

[12] W. Rudin, Functional Analysis, $2^{\text {nd }}$ edition, McGraw-Hill, New York 1991.

[13] W. Rudin, Real and Complex Analysis, $3^{\text {rd }}$ edition, McGraw-Hill, New York 1987.

[14] J. Westwater, On Edwards model for polymer chains, in Trends and Developments in the Eighties (S. Albeverio and P. Blanchard, eds.), Bielefeld Encounters in Math. Phys. 4/5. World Scientific, Singapore 1984. 